\title{
ABJM Wilson loops in arbitrary representations
}

\author{
Yasuyuki Hatsuda, ${ }^{a, b}$ Masazumi Honda, ${ }^{c, d}$ Sanefumi Moriyama ${ }^{d, e}$ and \\ Kazumi Okuyama ${ }^{f}$ \\ ${ }^{a}$ DESY Theory Group, DESY Hamburg, \\ Notkestrasse 85, D-22603 Hamburg, Germany \\ ${ }^{b}$ Department of Physics, Tokyo Institute of Technology, \\ 2-12-1 Ookayama, Meguro-ku, Tokyo 152-8551, Japan \\ ${ }^{c}$ High Energy Accelerator Research Organization (KEK), \\ 1-1 Oho, Tsukuba, Ibaraki 305-0801, Japan \\ ${ }^{d}$ Yukawa Institute for Theoretical Physics, Kyoto University, \\ Kitashirakawa Oiwakecho, Sakyo-ku, Kyoto 606-8502, Japan \\ ${ }^{e}$ Kobayashi Maskawa Institute and Graduate School of Mathematics, \\ Furocho, Chikusa-ku, Nagoya 464-8602, Japan \\ ${ }^{f}$ Department of Physics, Shinshu University, \\ 3-1-1 Asahi, Matsumoto 390-8621, Japan \\ E-mail: yasuyuki.hatsuda@desy.de, mhonda@post.kek.jp, \\ moriyama@math.nagoya-u.ac.jp, kazumi@azusa.shinshu-u.ac.jp
}

ABSTRACT: We study vacuum expectation values (VEVs) of circular half BPS Wilson loops in arbitrary representations in ABJM theory. We find that those in hook representations are reduced to elementary integrations thanks to the Fermi gas formalism, which are accessible from the numerical studies similar to the partition function in the previous studies. For non-hook representations, we show that the VEVs in the grand canonical formalism can be exactly expressed as determinants of those in the hook representations. Using these facts, we can study the instanton effects of the VEVs in various representations. Our results are consistent with the worldsheet instanton effects studied from the topological string and a prescription to include the membrane instanton effects by shifting the chemical potential, which has been successful for the partition function.

Keywords: Matrix Models, Wilson, 't Hooft and Polyakov loops, Topological Strings, M-Theory

ARXIV EPRINT: 1306.4297 


\section{Contents}

1 Introduction 1

2 BPS Wilson loops in general representations 5

2.1 Partition function 5

$\begin{array}{ll}2.2 & \text { Operator insertion }\end{array}$

3 Half BPS Wilson loops I: hook representations 9

3.1 Representations of the superalgebra 9

$\begin{array}{ll}3.2 & \text { Beyond winding Wilson loops }\end{array}$

$\begin{array}{ll}3.3 & \text { Single-hook representations } \\ \end{array}$

4 Half BPS Wilson loops II: general representations 11

$\begin{array}{lll}4.1 & \text { Non-hook representations } & 11\end{array}$

$\begin{array}{ll}4.2 \text { A proof } & 12\end{array}$

$\begin{array}{lll}4.3 & \text { Fermionic representation } & 15\end{array}$

5 Relation to open topological strings $\quad \mathbf{1 5}$

$\begin{array}{lll}5.1 & \text { Perturbative part } & 17\end{array}$

$\begin{array}{lll}5.2 \text { Worldsheet instantons } & 18\end{array}$

6 Numerical study and membrane instantons $\quad 19$

$\begin{array}{lll}6.1 \text { A procedure } & 20\end{array}$

6.2 Fundamental representation 22

6.3 Young diagrams with two boxes 23

6.4 Young diagrams with three boxes 24

6.5 Young diagrams with four boxes 24

$\begin{array}{lll}6.6 & \text { Implications } & 25\end{array}$

$\begin{array}{llr}7 & \text { Conclusion } & 26\end{array}$

\section{Introduction}

Recently, there has been much progress in understanding membranes in M-theory. It was proposed in [1] that the low energy effective theory on the $N$ multiple M2-branes on the geometry $\mathbb{C}^{4} / \mathbb{Z}_{k}$ is described by the 3 -dimensional $\mathcal{N}=6$ supersymmetric generalization of the Chern-Simons matter theory with gauge group $\mathrm{U}(N)_{k} \times \mathrm{U}(N)_{-k}$ commonly referred to as ABJM theory. Furthermore it has been shown by using the localization technique [2] that a class of supersymmetric observables in the ABJM theory on $S^{3}$ are described by so-called ABJM matrix model [3-6]. 
The partition function $Z(N)$ is the first fundamental quantity to be studied. After the rather standard matrix model analysis in [7-9], there appeared a seminal paper, which rewrites the ABJM partition function into the partition function of an ideal Fermi gas system [10] (see also [11-13]). One of the advantages in this Fermi gas formalism is that instead of the stringy 't Hooft expansion, we can access to the M-theory region directly by taking large $N$ limit with $k$ fixed. As is usual in the statistical system, instead of the partition function, it is convenient to define the grand partition function

$$
\Xi(z)=\sum_{N=0}^{\infty} z^{N} Z(N),
$$

by introducing the fugacity $z=e^{\mu}$ with the chemical potential $\mu$. Subsequently in [1421], the partition function of the ABJM theory was studied extensively from this grand partition function of the Fermi gas system. Finally, it turned out that the grand potential $J(\mu)=\log \Xi(z)$ can be separated into the perturbative, worldsheet instanton [22], membrane instanton $[8,23]$ and bound state part. The worldsheet instanton part is determined directly from the topological string result [18]. The membrane instanton part is also related to the refined topological string [21]. As found in [20], the contributions from all of the bound states can be incorporated to the worldsheet instanton effects by shifting the chemical potential $\mu$ to an "effective" chemical potential $\mu_{\text {eff }}$, which is described by the sum of $\mu$ and a part of the pure membrane instanton effects.

Here we proceed to study the second fundamental quantity, namely, the vacuum expectation value (VEV) of the circular half BPS Wilson loop ${ }^{1}$ firstly introduced in $[24,25]$. The half BPS Wilson loops have nice counterparts in the open topological string, as was pointed out in $[7,25]$. This is one of our motivation that we focus on them here. The half BPS Wilson loops are classified by representations $\mathbf{R}$ of the supergroup $\mathrm{U}(N \mid N)$, which includes the gauge group $\mathrm{U}(N) \times \mathrm{U}(N)$ as the bosonic subgroup. By using the localization method [3-6], the unnormalized VEV of the Wilson loop $W_{\mathbf{R}}$ in the representation $\mathbf{R}$ is written as

$$
\begin{aligned}
\left\langle W_{\mathbf{R}}\right\rangle_{N} & =\frac{1}{(N !)^{2}} \int \prod_{i} \frac{d \mu_{i}}{2 \pi} \frac{d \nu_{i}}{2 \pi} \frac{\prod_{i<j}\left(2 \sinh \frac{\mu_{i}-\mu_{j}}{2}\right)^{2}\left(2 \sinh \frac{\nu_{i}-\nu_{j}}{2}\right)^{2}}{\prod_{i, j}\left(2 \cosh \frac{\mu_{i}-\nu_{j}}{2}\right)^{2}} e^{-\frac{1}{2 g_{s}} \sum_{i}\left(\mu_{i}^{2}-\nu_{i}^{2}\right)} \operatorname{Str}_{\mathbf{R}} U \\
U & =\left(\begin{array}{cc}
U_{\mu} & 0 \\
0 & -U_{\nu}
\end{array}\right), \quad U_{\mu}=\operatorname{diag}\left(e^{\mu_{i}}\right), \quad U_{\nu}=\operatorname{diag}\left(e^{\nu_{i}}\right)
\end{aligned}
$$

where $g_{s}=\frac{2 \pi i}{k}$ is the coupling constant, and $\operatorname{Str}_{\mathbf{R}}$ is the $\mathrm{U}(N \mid N)$ character in the representation $\mathbf{R}$. A prescription to obtain $\operatorname{Str}_{\mathbf{R}}$ is summarized as follows. First, a representation of the supergroup $\mathrm{U}(N \mid N)$ is characterized by the super Young diagram, which has the same form as the usual Young diagram of the bosonic group $\mathrm{U}(\infty)$ (for example, see [26]). Then, the supertrace $\operatorname{Str}_{\mathbf{R}} U$ of the supergroup $\mathrm{U}(N \mid N)$ is found if we formally replace the power sum $\operatorname{tr} U^{n}$ in $\operatorname{tr}_{\mathbf{R}} U$ of $\mathrm{U}(\infty)$ by $\operatorname{Str} U^{n}$. Note that $U$ appearing $\operatorname{in}_{\operatorname{Str}_{\mathbf{R}}} U$ is a $2 N \times 2 N$ matrix defined by (1.2).

\footnotetext{
${ }^{1}$ Below we often refer to this circular half BPS Wilson loop simply as "the half BPS Wilson loop". As seen later in (1.2), after applying the localization method, the Wilson loop operator becomes a character of a certain group representation in mathematical terminology, to which we mostly continue to refer as the Wilson loop by a slight abuse of terminology.
} 


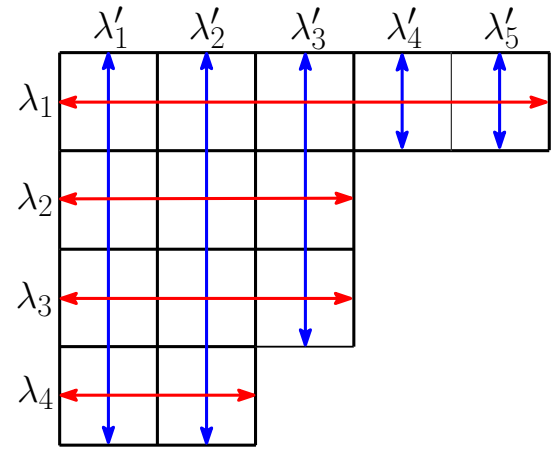

(a) Partition notation

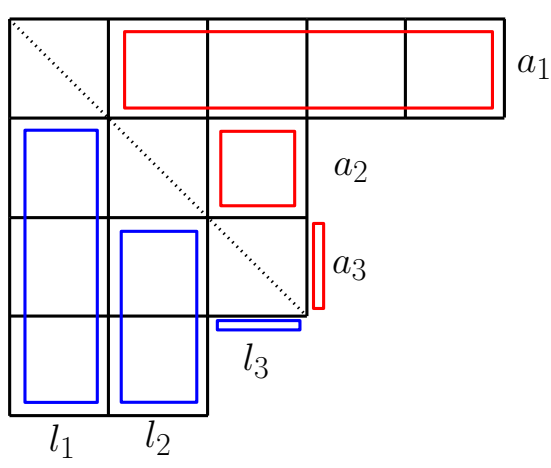

(b) Frobenius notation

Figure 1. (a) The partition notation $\left[\lambda_{1} \lambda_{2} \lambda_{3} \cdots\right]$ with its transpose $\left[\lambda_{1}^{\prime} \lambda_{2}^{\prime} \lambda_{3}^{\prime} \cdots\right]$ and (b) the Frobenius notation $\left(a_{1} a_{2} \cdots a_{r} \mid l_{1} l_{2} \cdots l_{r}\right)$ for the same Young diagram. Here $r=\max \left\{s \mid \lambda_{s}-s \geq\right.$ $0\}=\max \left\{s \mid \lambda_{s}^{\prime}-s \geq 0\right\}$ is the number of diagonal boxes, and $a_{p}, l_{q}$ denote the horizontal and vertical distances from each diagonal box, respectively, given by $a_{p}=\lambda_{p}-p, l_{q}=\lambda_{q}^{\prime}-q$. In the above case, the Young diagram is $\left[\lambda_{1} \lambda_{2} \lambda_{3} \lambda_{4}\right]=[5,3,3,2]$ in the partition notation with its transpose $\left[\lambda_{1}^{\prime} \lambda_{2}^{\prime} \lambda_{3}^{\prime} \lambda_{4}^{\prime} \lambda_{5}^{\prime}\right]=[4,4,3,1,1]$, while it is $\left(a_{1} a_{2} a_{3} \mid l_{1} l_{2} l_{3}\right)=(4,1,0 \mid 3,2,0)$ in the Frobenius notation.

The computation of the VEVs using the Fermi gas formalism was initiated in [27], where the inserted observables are restricted to the operators with winding number $n$, $\operatorname{Str} U^{n}$. Very recently, it was proposed in [28] that it is possible to study the perturbative part and the worldsheet instanton part using the topological strings. This subject keeps on attracting various studies. ${ }^{2}$

In this paper, we present a Fermi gas formalism for the VEVs in arbitrary representations, suitable for numerical study, and study these non-perturbative effects. As in the partition function, besides the worldsheet instanton contribution, we also find the contribution coming from the membrane instanton, which is difficult to be known from the topological string theory. In the following of this introduction, we would like to explain our results in more details. Just as in the partition function, it is useful to consider the VEV in the grand canonical ensemble defined by

$$
\left\langle W_{\mathbf{R}}\right\rangle^{\mathrm{GC}}=\frac{1}{\Xi(z)} \sum_{N=0}^{\infty} z^{N}\left\langle W_{\mathbf{R}}\right\rangle_{N} .
$$

Note that once we know $\left\langle W_{\mathbf{R}}\right\rangle^{\mathrm{GC}}$, the VEVs in the canonical ensemble is easily recovered.

First, we find a formula for the VEV of the Wilson loop in the hook representation ${ }^{3}$ $\mathbf{R}=(a \mid l)$ in terms of a certain convolution of integrations

$$
\left\langle W_{(a \mid l)}\right\rangle^{\mathrm{GC}}=\left\langle a\left|\frac{z}{1+z \rho_{1}}\right| l\right\rangle .
$$

\footnotetext{
${ }^{2}$ See, for example, [29] for perturbative studies of the Wilson loop VEVs, [30, 31] for the holographic studies, [31-33] for generalizations of contour and [34] for more general Chern-Simons matter theory.

${ }^{3}$ Throughout this paper, we use the Frobenius notation to express representations of $\mathrm{U}(N \mid N)$ illustrated in figure 1 .
} 
Here $\rho_{1}$ is the density operator of the Fermi-gas system defined later in (2.9), and the states $\langle a|$ and $|l\rangle$ in the coordinate basis are given by (3.9). The expression (1.4) is accessible from the numerical studies with very high precision.

Second, we extend our analysis to general representation $\mathbf{R}=\left(a_{1} a_{2} \cdots a_{r} \mid l_{1} l_{2} \cdots l_{r}\right)$. In operator level, the Wilson loop is simply given by the determinant of those in the hook representations, known as the Giambelli formula, ${ }^{4}$

$$
W_{\left(a_{1} a_{2} \cdots a_{r} \mid l_{1} l_{2} \cdots l_{r}\right)}\left(e^{\mu}, e^{\nu}\right)=\operatorname{det}_{p, q} W_{\left(a_{p} \mid l_{q}\right)}\left(e^{\mu}, e^{\nu}\right) .
$$

In this paper, we find that the VEVs in the grand canonical ensemble exactly satisfy the same type of the formula,

$$
\left\langle W_{\left(a_{1} a_{2} \cdots a_{r} \mid l_{1} l_{2} \cdots l_{r}\right)}\right\rangle^{\mathrm{GC}}=\left\langle\operatorname{det}_{p, q} W_{\left(a_{p} \mid l_{q}\right)}\right\rangle^{\mathrm{GC}}=\operatorname{det}_{p, q}\left(\left\langle W_{\left(a_{p} \mid l_{q}\right)}\right)^{\mathrm{GC}}\right) .
$$

Hence the VEVs of the half BPS Wilson loops in general representations can be computed only from those in hook representations. We emphasize that this result is very unexpected and non-trivial. In the mathematical context, a normalized linear functional $\langle\mathcal{O}\rangle$ of symmetric functions $\mathcal{O}$ satisfying the above property is called Giambelli compatible (see e.g. [35]). Let us further call a linear functional being factorizable if it satisfies the property $\left\langle\mathcal{O}_{1} \mathcal{O}_{2}\right\rangle=\left\langle\mathcal{O}_{1}\right\rangle\left\langle\mathcal{O}_{2}\right\rangle$. Note that the factorizability implies the Giambelli compability. In this terminology, we show that the grand canonical VEV of the half BPS Wilson loop is Giambelli compatible but not factorizable. We also find that its perturbative part is factorizable (see (5.10)). Note that the factorization of the grand canonical VEV also implies that of the canonical VEV in the large $N$ limit, which is natural from the physical viewpoint. The factorization property, however, is generically broken by the instanton contributions. Nevertheless, the Giambelli compatibility is still preserved after the instanton effects are taken into account.

Finally, using our results (1.4) and (1.6), we also study the structure of the instanton corrections to the VEVs in various representations by the numerical studies. The VEVs, in general, receive the following corrections,

$$
\left\langle W_{\mathbf{R}}\right\rangle^{\mathrm{GC}}=W_{\mathbf{R}}^{\mathrm{GC}(\text { pert })}(\mu, k)\left(1+W_{\mathbf{R}}^{\mathrm{GC}(\mathrm{WS})}(\mu, k)+W_{\mathbf{R}}^{\mathrm{GC}(\text { others })}(\mu, k)\right),
$$

where $W_{\mathbf{R}}^{\mathrm{GC}(\text { pert })}(\mu, k)$ is the perturbative part, $W_{\mathbf{R}}^{\mathrm{GC}(\mathrm{WS})}(\mu, k)$ is the worldsheet instanton correction, and $W_{\mathbf{R}}^{\mathrm{GC}(\mathrm{others})}(\mu, k)$ consists of the pure membrane instanton correction and the contribution from the bound states. We have found that our numerical results match with the topological string prediction of the perturbative part and the worldsheet instanton part with the chemical potential shifted from $\mu$ to $\mu_{\text {eff }}$ to incorporate the contribution from the membrane instantons and the bound states:

$$
\left\langle W_{\mathbf{R}}\right\rangle^{\mathrm{GC}}=W_{\mathbf{R}}^{\mathrm{GC}(\text { pert })}\left(\mu_{\mathrm{eff}}, k\right)\left(1+W_{\mathbf{R}}^{\mathrm{GC}(\mathrm{WS})}\left(\mu_{\mathrm{eff}}, k\right)\right),
$$

exactly the same as in the partition function. Here the "effective" chemical potential $\mu_{\text {eff }}$ was introduced in [20] in order to explain the bound state contribution in the grand

\footnotetext{
${ }^{4}$ We often write $W_{\mathbf{R}}\left(e^{\mu}, e^{\nu}\right)$ instead of $\operatorname{Str}_{\mathbf{R}} U$ to represent the Wilson loop insertion apparently.
} 
potential,

$$
\mu_{\mathrm{eff}}=\mu+\frac{\pi^{2} k}{2} \sum_{\ell=1}^{\infty} a_{\ell}(k) e^{-2 \ell \mu},
$$

where $a_{\ell}(k)$ are the functions appearing in the membrane instanton correction in the grand potential. The forms of $a_{\ell}(k)$ are exactly computed by the refined topological string on local $\mathbb{P}^{1} \times \mathbb{P}^{1}[21]$. We should stress that the perturbative part and the worldsheet instanton part in (1.8) are computed from the open topological string on local $\mathbb{P}^{1} \times \mathbb{P}^{1}$ as we will see in section 5 . Thus our result states that once we determine the topological string free energy on this background, we can exactly find the VEVs of the half BPS Wilson loops in general representations in the ABJM theory.

The organization of this paper is as follows. In the next section we present a general framework to study the VEVs of the BPS Wilson loops and apply it to the half BPS case in the hook representation in section 3. Since it is difficult to apply this formalism directly to the half BPS Wilson loops in the non-hook representation, we shall present an alternative method in section 4, which works only for the half BPS Wilson loop. After reviewing the results from the topological strings in section 5 , we summarize our numerical study in section 6 . Finally we conclude in section 7 .

\section{BPS Wilson loops in general representations}

Here we present methods to study the VEV of the Wilson loop in the ABJM theory using the Fermi gas formalism. We shall first present a framework to study general 1/6 BPS Wilson loop constructed in [36-38], which includes the half BPS Wilson loop as a special case.

\subsection{Partition function}

For this purpose let us first review the derivation of the Fermi gas formalism for the partition function [10] carefully because our Wilson loop insertion is based heavily on it. The starting point is the partition function of the ABJM matrix model [3-6]:

$$
Z(N)=\frac{1}{(N !)^{2}} \int \prod_{i} \frac{d \mu_{i}}{2 \pi} \frac{d \nu_{i}}{2 \pi} \frac{\prod_{i<j}\left(2 \sinh \frac{\mu_{i}-\mu_{j}}{2}\right)^{2}\left(2 \sinh \frac{\nu_{i}-\nu_{j}}{2}\right)^{2}}{\prod_{i, j}\left(2 \cosh \frac{\mu_{i}-\nu_{j}}{2}\right)^{2}} e^{-\frac{1}{2 g_{s}}\left(\sum_{i} \mu_{i}^{2}-\sum_{j} \nu_{j}^{2}\right)}
$$

By using the Cauchy identity and performing a Fourier transformation, the partition function (2.1) is rewritten into

$$
\begin{aligned}
Z(N)= & \frac{1}{N !} \int \prod_{i} \frac{d x_{i}}{\hbar} \frac{d y_{i}}{\hbar} \sum_{\sigma \in S_{N}}(-1)^{\sigma} \\
& \times \int \prod_{i} \frac{d p_{i}}{2 \pi} \frac{d q_{i}}{2 \pi} \prod_{i}\left[\frac{e^{-\frac{i}{\hbar} p_{i}\left(x_{i}-y_{i}\right)}}{2 \cosh \frac{p_{i}}{2}} \frac{\left.e^{-\frac{i}{\hbar} q_{i}\left(x_{i}-y_{\sigma}-1(i)\right.}\right)}{2 \cosh \frac{q_{i}}{2}}\right] e^{\frac{i}{2 \hbar}\left(\sum_{i} x_{i}^{2}-\sum_{j} y_{j}^{2}\right)},
\end{aligned}
$$


where we rescale the integration variables as $\mu_{i}=\frac{x_{i}}{k}, \nu_{j}=\frac{y_{j}}{k}$ and $\hbar=2 \pi k$. After performing the Gaussian integral over $x$ and $y$ by completing the square in the exponent

$$
\begin{aligned}
& \frac{i}{2 \hbar} x_{i}^{2}-\frac{i}{\hbar} x_{i}\left(p_{i}+q_{i}\right)-\frac{i}{2 \hbar} y_{i}^{2}+\frac{i}{\hbar} y_{i}\left(p_{i}+q_{\sigma(i)}\right) \\
& \quad=\frac{i}{2 \hbar}\left(x_{i}-p_{i}-q_{i}\right)^{2}-\frac{i}{2 \hbar}\left(y_{i}-p_{i}-q_{\sigma(i)}\right)^{2}-\frac{i}{2 \hbar}\left(p_{i}+q_{i}\right)^{2}+\frac{i}{2 \hbar}\left(p_{i}+q_{\sigma(i)}\right)^{2}
\end{aligned}
$$

and noting the cancellation of the $p^{2}$ and $q^{2}$ terms, the partition function becomes

$$
Z(N)=\frac{1}{N !} \int \prod_{i} \frac{d p_{i} d q_{i}}{2 \pi \hbar} \sum_{\sigma \in S_{N}}(-1)^{\sigma} \prod_{i} \frac{e^{i p_{i}\left(q_{\sigma(i)}-q_{i}\right) / \hbar}}{2 \cosh \frac{p_{i}}{2} \cdot 2 \cosh \frac{q_{i}}{2}} .
$$

If we further integrate over $p$ in (2.4), then we find

$$
Z(N)=\frac{1}{N !} \int \prod_{i} \frac{d q_{i}}{\hbar} \sum_{\sigma \in S_{N}}(-1)^{\sigma} \prod_{i} \frac{1}{\sqrt{2 \cosh \frac{q_{\sigma(i)}}{2}}} \frac{1}{2 \cosh \frac{q_{\sigma(i)}-q_{i}}{2 k}} \frac{1}{\sqrt{2 \cosh \frac{q_{i}}{2}}} .
$$

Since the partition function $Z(N)$ has the form of an ideal Fermi gas system as

$$
Z(N)=\frac{1}{N !} \sum_{\sigma \in S_{N}}(-1)^{\sigma} \int \prod_{i} \frac{d q_{i}}{\hbar} \prod_{i} \rho_{1}\left(q_{i}, q_{\sigma(i)}\right),
$$

with

$$
\rho_{1}\left(q_{i}, q_{j}\right)=\frac{1}{\sqrt{2 \cosh \frac{q_{j}}{2}}} \frac{1}{2 \cosh \frac{q_{j}-q_{i}}{2 k}} \frac{1}{\sqrt{2 \cosh \frac{q_{i}}{2}}},
$$

it is easier to consider the grand canonical partition function (1.1) by introducing the fugacity $z=e^{\mu}$. One can show that the grand partition function is expressed as a Fredholm determinant,

$$
\Xi(z)=\operatorname{Det}\left(1+z \rho_{1}\right)
$$

where the determinant Det is taken over the whole Hilbert space of the Fermi gas system. In the operator formalism, the density matrix $\rho_{1}$ is given by

$$
\rho_{1}=\sqrt{Q} P \sqrt{Q}, \quad \text { with } \quad P=\frac{1}{2 \cosh \frac{p}{2}}, Q=\frac{1}{2 \cosh \frac{q}{2}},
$$

where $q$ and $p$ satisfy the canonical commutation relation $[q, p]=i \hbar$ with $\hbar=2 \pi k$. We adopt this notation in what follows.

\subsection{Operator insertion}

General 1/6 BPS Wilson loops in the ABJM theory are generated by the following type of operator [3]:

$$
\prod_{i} f\left(e^{\mu_{i}}\right) g\left(e^{\nu_{i}}\right)
$$


where $f(x)$ and $g(x)$ are functions of $x$. In this section we translate the insertion of this operator into the one of a certain quantum mechanical operator expressed by $(q, p)$.

As a warm up, let us first consider the operator insertion

$$
e^{n \mu_{M}}=e^{\frac{2 \pi n x_{M}}{\hbar}},
$$

into the partition function (2.2). After completing the square in integrating over $x_{M}$ and combining with the contribution from integrating $y_{M}$ as in the computation of the partition function, we find an extra contribution into the exponent:

$$
\frac{i}{\hbar} p_{M}(-2 \pi i n)+\frac{2 \pi n}{\hbar}\left(q_{M}+\pi i n\right)
$$

Performing the integration over $p_{M}$, the unnormalized VEV is finally given by

$$
\begin{aligned}
\left\langle e^{n \mu_{M}}\right\rangle_{N}=\frac{1}{N !} & \int \prod_{i} \frac{d q_{i}}{\hbar} \sum_{\sigma \in S_{N}}(-1)^{\sigma} \prod_{i \neq M} \rho_{1}\left(q_{\sigma(i)}, q_{i}\right) \\
& \times \frac{1}{\sqrt{2 \cosh \frac{q_{\sigma(M)}}{2}}} \frac{e^{\frac{2 \pi n}{\hbar}\left(q_{M}+\pi i n\right)}}{2 \cosh \frac{q_{\sigma(M)}-q_{M}-2 \pi i n}{2 k}} \frac{1}{\sqrt{2 \cosh \frac{q_{M}}{2}}} .
\end{aligned}
$$

In the language of quantum mechanical operators, the second line can be interpreted as the matrix element

$$
\left\langle q_{\sigma(M)}\left|\sqrt{Q} P e^{\frac{n(q+p)}{k}} \sqrt{Q}\right| q_{M}\right\rangle=\left\langle q_{\sigma(M)}\left|\sqrt{Q} e^{\frac{n p}{k}} P e^{\frac{n(q+i \pi n)}{k}} \sqrt{Q}\right| q_{M}\right\rangle .
$$

Therefore we conclude that the insertion of the operator $e^{n \mu_{M}}$ amounts to the insertion of the operator $W^{n}$ to the right of $P$, where $W$ is defined by

$$
W=e^{\frac{q+p}{k}} .
$$

Similarly, we find that the insertion of the operator $e^{n \nu_{M}}$ amounts to insertion of the same operator $W^{n}$ to the left of $P$. This can be seen by repeating the square completion in the exponent with an extra factor

$$
\frac{i}{\hbar} p_{M}(-2 \pi i n)+\frac{2 \pi n}{\hbar}\left(q_{\sigma(M)}-\pi i n\right)
$$

and computing of the matrix element

$$
\left\langle q_{\sigma(i)}\left|\sqrt{Q} e^{\frac{n(q+p)}{k}} P \sqrt{Q}\right| q_{i}\right\rangle=\left\langle q_{\sigma(i)}\left|\sqrt{Q} e^{\frac{n(q-\pi i n)}{k}} e^{\frac{n p}{k}} P \sqrt{Q}\right| q_{i}\right\rangle .
$$

Note that this interpretation is factor-wise. Namely, not only other additive terms in the insertion do not affect this interpretation, but this interpretation is valid even if this operator is multiplied by other operators. We can also see that the simultaneous insertion at the same position $M$, namely, $e^{m \mu_{M}+n \nu_{M}}$ also works well.

Therefore we can summarize the computation rule as follows. For the case of the partition function, we finally end up with the summation over the conjugacy classes and the study of

$$
\operatorname{Tr} \rho_{1}^{m}=\operatorname{Tr} \sqrt{Q} P Q P Q P Q P \cdots \sqrt{Q}
$$


For the case of Wilson loop, we insert $W$ into various slots between $Q$ and $P$ in this trace. The insertion pattern depends on the representation, but since we are considering the gauge invariant operator, we have to take a trace, namely, sum over all the insertion slots. Hence our formula can be summarized as

$$
\Xi(z)\left\langle\prod_{i} f\left(e^{\mu_{i}}\right) g\left(e^{\nu_{i}}\right)\right\rangle^{\mathrm{GC}}=\operatorname{Det}(1+z \sqrt{Q} g(W) P f(W) \sqrt{Q}),
$$

where $\langle\mathcal{O}\rangle^{\mathrm{GC}}$ denotes the expectation value of the operator $\mathcal{O}$ in the grand canonical ensemble (1.3). Once the grand canonical VEV is understood, one can easily return to the canonical VEV via

$$
\langle\mathcal{O}\rangle_{N}=\frac{1}{2 \pi i} \oint \frac{d z}{z^{N+1}} \Xi(z)\langle\mathcal{O}\rangle^{\mathrm{GC}} .
$$

Alternatively, we can show the relation (2.19) using the operator formalism as follows. The expectation value of $\prod_{i} f\left(e^{\mu_{i}}\right) g\left(e^{\nu_{i}}\right)$ at fixed $N$ is given by

$$
\begin{aligned}
& \left\langle\prod_{i} f\left(e^{\mu_{i}}\right) g\left(e^{\nu_{i}}\right)\right\rangle_{N}=\frac{1}{N !} \sum_{\sigma \in S_{N}}(-1)^{\sigma} \int \prod_{i} \frac{d \mu_{i}}{2 \pi} \frac{d \nu_{i}}{2 \pi} \\
& \quad \times \prod_{i} f\left(e^{\mu_{i}}\right) g\left(e^{\nu_{i}}\right) e^{\frac{i k}{4 \pi}\left(\mu_{i}^{2}-\nu_{i}^{2}\right)} \prod_{i} \frac{1}{2 \cosh \frac{\nu_{\sigma(i)}-\mu_{i}}{2}} \frac{1}{2 \cosh \frac{\mu_{i}-\nu_{i}}{2}} .
\end{aligned}
$$

By rescaling $\mu_{i}=\frac{x_{i}}{k}, \nu_{i}=\frac{y_{i}}{k}$, this is rewritten as

$$
\left\langle\prod_{i} f\left(e^{\mu_{i}}\right) g\left(e^{\nu_{i}}\right)\right\rangle_{N}=\frac{1}{N !} \sum_{\sigma \in S_{N}}(-1)^{\sigma} \int \prod_{i} \frac{d y_{i}}{\hbar} \prod_{i} \rho\left(y_{i}, y_{\sigma(i)}\right),
$$

where $\rho$ denotes the density matrix in the presence of operator insertion

$$
\begin{aligned}
\rho\left(y_{i}, y_{j}\right) & =\int \frac{d x}{\hbar} \frac{e^{\frac{i}{2 \hbar}\left(x^{2}-y_{j}^{2}\right)} f\left(e^{\frac{x}{k}}\right) g\left(e^{\frac{y_{j}}{k}}\right)}{2 \cosh \frac{y_{j}-x}{2 k} \cdot 2 \cosh \frac{x-y_{i}}{2 k}} \\
& =\int \frac{d x}{\hbar}\left\langle y_{j}\left|e^{-\frac{i q^{2}}{2 \hbar}} g\left(e^{\frac{q}{k}}\right) \frac{1}{2 \cosh \frac{p}{2}}\right| x\right\rangle\left\langle x\left|f\left(e^{\frac{q}{k}}\right) e^{\frac{i q^{2}}{2 \hbar}} \frac{1}{2 \cosh \frac{p}{2}}\right| y_{i}\right\rangle \\
& =\left\langle y_{j}\left|e^{-\frac{i q^{2}}{2 \hbar}} g\left(e^{\frac{q}{k}}\right) \frac{1}{2 \cosh \frac{p}{2}} f\left(e^{\frac{q}{k}}\right) e^{\frac{i q^{2}}{2 \hbar}} \frac{1}{2 \cosh \frac{p}{2}}\right| y_{i}\right\rangle .
\end{aligned}
$$

This can be written as an operator equation

$$
\begin{aligned}
\rho & =e^{-\frac{i q^{2}}{2 \hbar}} g\left(e^{\frac{q}{k}}\right) \frac{1}{2 \cosh \frac{p}{2}} f\left(e^{\frac{q}{k}}\right) e^{\frac{i q^{2}}{2 \hbar}} \frac{1}{2 \cosh \frac{p}{2}} \\
& =e^{-\frac{i q^{2}}{2 \hbar}} e^{-\frac{i p^{2}}{2 \hbar}} g\left(e^{\frac{q+p}{k}}\right) \frac{1}{2 \cosh \frac{p}{2}} f\left(e^{\frac{q+p}{k}}\right) \frac{1}{2 \cosh \frac{q}{2}} e^{\frac{i p^{2}}{2 \hbar}} e^{\frac{i q^{2}}{2 \hbar}},
\end{aligned}
$$

where we have used

$$
e^{\frac{i q^{2}}{2 \hbar}} F(p) e^{-\frac{i q^{2}}{2 \hbar}}=F(p-q), \quad e^{\frac{i p^{2}}{2 \hbar}} G(q) e^{-\frac{i p^{2}}{2 \hbar}}=G(q+p) .
$$

Therefore, up to a similarity transformation the density matrix in (2.24) becomes

$$
\rho=\sqrt{Q} g(W) P f(W) \sqrt{Q}
$$

which reproduces (2.19). 


\section{Half BPS Wilson loops I: hook representations}

In the previous section, we have presented a general framework to study the VEVs of the general 1/6 BPS Wilson loop in the Fermi gas formalism. Especially we have reduced the problem into computing the trace with alternating operators $Q$ and $P$ and various $W$-insertions. This quantity, however, is still difficult to compute, at least, numerically with high precision. Here we would like to see what kind of simplification will occur if we restrict ourselves to the half BPS Wilson loops.

\subsection{Representations of the superalgebra}

The half BPS Wilson loop is classified by the representation of $\mathrm{U}(N \mid N)[24,25]$. In this subsection we review representations of the supergroup $\mathrm{U}(N \mid N)$. For this purpose, it is convenient to consider representations of $\mathrm{U}(\infty)$. A simple prescription to derive the character of $\mathrm{U}(N \mid N)$ is to formally replace $\operatorname{tr} U^{n}$ in the character $\operatorname{tr}_{\mathbf{R}} U$ of $\mathrm{U}(\infty)$ by $\operatorname{Str} U^{n}$ :

$$
\operatorname{Str}_{\mathbf{R}} U=\left.\operatorname{tr}_{\mathbf{R}} U\right|_{\operatorname{tr} U^{n} \rightarrow \operatorname{Str} U^{n}} .
$$

Note that the character $\operatorname{tr}_{\mathbf{R}} U$ is given by the Schur function associated with the Young diagram $\mathbf{R}$. The supertrace $\operatorname{Str}_{\mathbf{R}} U$ can be expressed by a combination of characters of two bosonic subgroups $\mathrm{U}(N)$ of $\mathrm{U}(N \mid N)$. For example, in the case of the 2nd anti-symmetric representation $(0 \mid 1)$, the superalgebraic generalization turns out to be

$$
\begin{aligned}
\operatorname{Str}_{(0 \mid 1)} U & =\frac{1}{2}(\operatorname{Str} U)^{2}-\frac{1}{2} \operatorname{Str} U^{2} \\
& =\operatorname{tr}_{(0 \mid 1)} U_{\mu}+\operatorname{tr}_{(0 \mid 0)} U_{\mu} \operatorname{tr}_{(0 \mid 0)} U_{\nu}+\operatorname{tr}_{(1 \mid 0)} U_{\nu}
\end{aligned}
$$

where $U_{\mu}$ and $U_{\nu}$ are the bosonic parts of $U$ (see (1.2)). Below, we often denote the supertrace $\operatorname{Str}_{\mathbf{R}} U$ by $W_{\mathbf{R}}\left(e^{\mu}, e^{\nu}\right)$, and use the abbreviation $W_{\mathbf{R}}=W_{\mathbf{R}}\left(e^{\mu}, e^{\nu}\right)$ as long as there is no risk of confusion.

\subsection{Beyond winding Wilson loops}

The Wilson loop with the winding number $n$

$$
\operatorname{Str} U^{n}=\sum_{i} e^{n \mu_{i}}-(-1)^{n} \sum_{i} e^{n \nu_{i}}
$$

was studied extensively in [27]. By revisiting this in our formalism, we will obtain a hint to study the more general representations as in the following.

In our formalism, applying the rule in (2.19) with the choice,

$$
f(W)=1+t W^{n}, \quad g(W)=\frac{1}{1+t(-W)^{n}}=\frac{1}{f(-W)},
$$

and picking up the linear term in $t$, the grand canonical VEV of $\operatorname{Str} U^{n}$ is given by

$$
\left\langle\operatorname{Str} U^{n}\right\rangle^{\mathrm{GC}}=\operatorname{Tr}\left[R(z) \sqrt{Q}\left(P W^{n}-(-W)^{n} P\right) \sqrt{Q}\right],
$$


with $R(z)$ defined by

$$
R(z)=\frac{z}{1+z \rho_{1}} .
$$

One can easily see that the operator appearing on the right-hand-side is expanded as

$$
P W^{n}-(-W)^{n} P=\sum_{l=0}^{n-1}(-1)^{l} W^{l}(W P+P W) W^{n-1-l} .
$$

Note that the operator appearing in the right-hand-side of (3.7) has the factorized form

$$
\left\langle q_{2}\left|W^{n}(W P+P W) W^{m}\right| q_{1}\right\rangle=\left\langle q_{2}\left|\frac{1}{\sqrt{Q}}\right| n\right\rangle\left\langle m\left|\frac{1}{\sqrt{Q}}\right| q_{1}\right\rangle,
$$

where the coordinate $q$ representations of $|n\rangle$ and $\langle m|$ are defined by

$$
\langle q \mid n\rangle=\frac{e^{\left(n+\frac{1}{2}\right) \frac{q}{k}-\frac{\pi i}{k} n(n+1)}}{\sqrt{2 \cosh \frac{q}{2}}}, \quad\langle m \mid q\rangle=\langle q \mid m\rangle^{*}=\frac{e^{\left(m+\frac{1}{2}\right) \frac{q}{k}+\frac{\pi i}{k} m(m+1)}}{\sqrt{2 \cosh \frac{q}{2}}} .
$$

As a formal operator relation, (3.8) is also written as

$$
\sqrt{Q} W^{n}(W P+P W) W^{m} \sqrt{Q}=|n\rangle\langle m| .
$$

Thus we finally obtain the grand canonical VEV of the winding Wilson loop (3.5) as

$$
\left\langle\operatorname{Str} U^{n}\right\rangle^{\mathrm{GC}}=\sum_{l=0}^{n-1}(-1)^{l}\langle n-1-l|R(z)| l\rangle .
$$

Comparing with the relation between the winding Wilson loop Str $U^{n}$ and the Wilson loop $W_{(a \mid l)}$ in the hook representation

$$
\operatorname{Str} U^{n}=\sum_{l=0}^{n-1}(-1)^{l} W_{(n-1-l \mid l)},
$$

it is tantalizing to expect the relation

$$
\left\langle W_{(a \mid l)}\right\rangle^{\mathrm{GC}}=\langle a|R(z)| l\rangle,
$$

which is true as we will see in the next subsection.

More generally, the computation of the VEVs of the half BPS operators reduces to picking up a certain function $f(W)$ and computing the Fredholm determinant of the corresponding density matrix $\rho_{f}$

$$
\Xi(z)\left\langle\prod_{i} \frac{f\left(e^{\mu_{i}}\right)}{f\left(-e^{\nu_{i}}\right)}\right\rangle^{\mathrm{GC}}=\operatorname{Det}\left(1+z \rho_{f}\right) \quad \text { with } \quad \rho_{f}=\sqrt{Q} \frac{1}{f(-W)} \operatorname{Pf}(W) \sqrt{Q} .
$$

Rewriting the density matrix in the above expression as

$$
\begin{aligned}
\rho_{f}-\rho_{1} & =\sqrt{Q} \frac{1}{f(-W)}(\operatorname{Pf}(W)-f(-W) P) \sqrt{Q} \\
& =\sqrt{Q} \frac{1}{f(-W)} \sum_{n=0}^{\infty} \frac{f^{(n)}(0)}{n !}\left(P W^{n}-(-W)^{n} P\right) \sqrt{Q},
\end{aligned}
$$

and recalling (3.7), one can see that the grand canonical VEV of the half BPS Wilson loops can always be written as a sum of the factorized functions. 


\subsection{Single-hook representations}

For the half BPS Wilson loop in a single-hook representation $(a \mid l)$, the generating function is given by [39]

$$
1+(s+t) \sum_{a, l=0}^{\infty} s^{a} t^{l} W_{(a \mid l)}=\operatorname{Sdet}\left(\frac{1+t U}{1-s U}\right)=\prod_{j=1}^{N} \frac{\left(1+t e^{\mu_{j}}\right)\left(1+s e^{\nu_{j}}\right)}{\left(1-s e^{\mu_{j}}\right)\left(1-t e^{\nu_{j}}\right)}
$$

When plugging

$$
f(W)=\frac{1+t W}{1-s W}
$$

into our formula (3.14), we find that the corresponding density matrix factorizes as

$$
\rho_{f}=\rho_{1}+(s+t) \sum_{a, l=0}^{\infty} s^{a} t^{l}|l\rangle\langle a| .
$$

Therefore, the grand canonical VEV of (3.16) becomes

$$
\begin{aligned}
& \left\langle 1+(s+t) \sum_{a, l=0}^{\infty} s^{a} t^{l} W_{(a \mid l)}\right\rangle^{\mathrm{GC}}=\frac{\operatorname{Det}\left(1+z \rho_{f}\right)}{\operatorname{Det}\left(1+z \rho_{1}\right)} \\
& =\operatorname{Det}\left(1+(s+t) \sum_{a, l=0}^{\infty} s^{a} t^{l} R(z)|l\rangle\langle a|\right)=1+(s+t) \sum_{a, l=0}^{\infty} s^{a} t^{l}\langle a|R(z)| l\rangle .
\end{aligned}
$$

Finally, the grand canonical VEV of $W_{(a \mid l)}$ is found to be (3.13) which is accessible from the numerical studies similar to the partition function in the previous studies $[16-18,20]$.

\section{Half BPS Wilson loops II: general representations}

In the previous sections, we have presented a method to compute the supersymmetric Wilson loops and shown that especially for the half BPS Wilson loop in the hook representation, there is a factorization, which at least simplifies the numerical study. The above analysis for the hook representation is, however, difficult to be extended to a general non-hook case. Here we shall present a completely different analysis which is effective for studying the non-hook representations from the hook representations but only suitable for the half BPS Wilson loop.

\section{$4.1 \quad$ Non-hook representations}

After understanding the VEV in the hook representation in the previous section, we can go beyond the hook representation step by step. Namely, we can substitute various functions for $f(W)$ and subtract the known hook part. For example, if we plug $f(W)=e^{t W}$, which corresponds to the generating function of $(\operatorname{Str} U)^{n}$, and compare $\mathcal{O}\left(t^{4}\right)$ terms, then we find

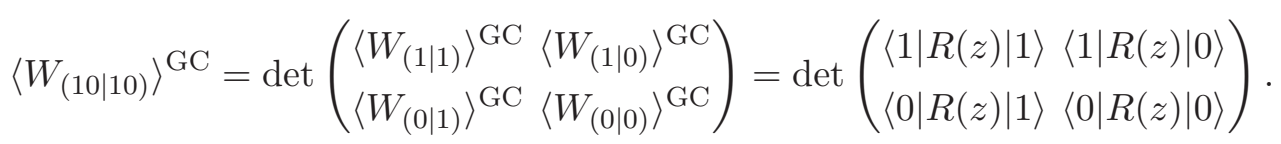


More generally, it is easy to imagine the expression in (1.6). By changing the function for $f(W)$, we will encounter various relations supporting this conjecture. However, it is difficult to prove it directly using this formulation.

\subsection{A proof}

Here we give a proof of (1.6):

$$
\left\langle W_{\left(a_{1} a_{2} \cdots a_{r} \mid l_{1} l_{2} \cdots l_{r}\right)}\right)^{\mathrm{GC}}=\operatorname{det}_{p, q}\left(\left\langle W_{\left(a_{p} \mid l_{q}\right)}\right\rangle^{\mathrm{GC}}\right),
$$

with a completely different method. ${ }^{5}$ The Giambelli formula states that

$$
W_{\left(a_{1} a_{2} \cdots a_{r} \mid l_{1} l_{2} \cdots l_{r}\right)}\left(e^{\mu}, e^{\nu}\right)=\operatorname{det}_{p, q} W_{\left(a_{p} \mid l_{q}\right)}\left(e^{\mu}, e^{\nu}\right) .
$$

Therefore, we would like to study

$$
\left\langle W_{\left(a_{1} a_{2} \cdots a_{r} \mid l_{1} l_{2} \cdots l_{r}\right)}\left(e^{\mu}, e^{\nu}\right)\right\rangle=\left\langle\operatorname{det}_{p, q} W_{\left(a_{p} \mid l_{q}\right)}\left(e^{\mu}, e^{\nu}\right)\right\rangle .
$$

Instead of computing it directly, here let us consider

$$
W(N)=\left\langle\operatorname{det}_{p, q}\left(\delta_{p, q}+t W_{\left(a_{p} \mid l_{q}\right)}\left(e^{\mu}, e^{\nu}\right)\right)\right\rangle,
$$

and pick up the coefficient of the highest $t^{r}$ term. The reason we want to consider $W(N)$ is because this is a generalization of the Cauchy determinant

$$
\frac{\operatorname{det}_{i, j}\left[\left(x_{i}+y_{j}\right)^{-1}+t \sum_{p=1}^{r} x_{i}^{a_{p}} y_{j}^{l_{p}}\right]}{\operatorname{det}_{i, j}\left[\left(x_{i}+y_{j}\right)^{-1}\right]}=\operatorname{det}_{p, q}\left[\delta_{p, q}+t W_{\left(a_{p} \mid l_{q}\right)}(x, y)\right] .
$$

The proof of this formula for $r=1$ is simply reduced to a more general formula in [40]. ${ }^{6}$ The proof for $r>1$ is reduced to the case of $r=1$ by the formula

$$
\operatorname{det}_{I, J=1, \cdots, N}\left(\delta_{I, J}+\sum_{k=1}^{n}(V)_{I k}\left(U^{\mathrm{T}}\right)_{k J}\right)=\operatorname{det}_{i, j=1, \cdots, n}\left(\delta_{i, j}+\sum_{K=1}^{N}\left(U^{\mathrm{T}}\right)_{i K}(V)_{K j}\right),
$$

which is true since $\operatorname{tr}\left(V U^{\mathrm{T}}\right)^{m}=\operatorname{tr}\left(U^{\mathrm{T}} V\right)^{m}$ for any positive integer $m$. To simplify our computation in the following, let us define

$$
\left[d \mu_{i}\right]=\frac{d \mu_{i}}{2 \pi} e^{-\frac{1}{2 g_{s}} \mu_{i}^{2}}, \quad\left[d \nu_{i}\right]=\frac{d \nu_{i}}{2 \pi} e^{\frac{1}{2 g_{s}} \nu_{i}^{2}} .
$$

\footnotetext{
${ }^{5}$ We are grateful to Sho Matsumoto for his collaborative contribution in sharing his idea of proof and the references with us in this subsection.

${ }^{6}$ The formula of [40] for the $r=1$ case is written as

$$
W_{(a \mid l)}(x, y)=\sum_{i, j=1}^{N} y_{i}^{l} M_{i j}^{-1} x_{j}^{a}
$$

where $M^{-1}$ is the inverse of Cauchy matrix $M_{i j}=1 /\left(x_{i}+y_{j}\right)$. One can show that the generating function of (4.5) reproduces (3.16).
} 
Then the quantity we want to compute becomes

$$
\begin{aligned}
W(N)= & \frac{1}{N !} \int \prod_{i}\left[d \mu_{i}\right]\left[d \nu_{i}\right] \operatorname{det}_{p, q}\left(\delta_{p, q}+t W_{\left(a_{p}, l_{q}\right)}\left(e^{\mu}, e^{\nu}\right)\right) \\
& \times \sum_{\sigma \in S_{N}}(-1)^{\sigma} \prod_{i} \frac{1}{2 \cosh \frac{\nu_{\sigma(i)}-\mu_{i}}{2}} \frac{1}{2 \cosh \frac{\mu_{i}-\nu_{i}}{2}} .
\end{aligned}
$$

Using the formula (4.4), we can rewrite this as

$$
W(N)=\frac{1}{N !} \sum_{\sigma \in S_{N}}(-1)^{\sigma} \int \prod_{i}\left[d \nu_{i}\right] \prod_{i} \boldsymbol{\rho}\left(\nu_{i}, \nu_{\sigma(i)}\right),
$$

where

$$
\boldsymbol{\rho}\left(\nu_{i}, \nu_{j}\right)=\int[d \mu]\left(\frac{1}{2 \cosh \frac{\nu_{j}-\mu}{2}}+t \sum_{p=1}^{r} e^{\left(l_{p}+1 / 2\right) \nu_{j}} e^{\left(a_{p}+1 / 2\right) \mu}\right) \frac{1}{2 \cosh \frac{\mu-\nu_{i}}{2}} .
$$

Since the VEV can be interpreted as the partition function of the ideal Fermi gas system just as the partition function (2.6), it is natural to introduce the generating function as

$$
\Omega(z)=\sum_{N=0}^{\infty} z^{N} W(N)=\operatorname{Det}(1+z \boldsymbol{\rho}),
$$

where Det is defined through the trace over the indices $\nu$ with the measure in (4.7). Therefore, if we define

$$
\begin{aligned}
\boldsymbol{Q}(\mu, \nu) & =\frac{1}{2 \cosh \frac{\mu-\nu}{2}}, & \boldsymbol{P}(\nu, \mu) & =\frac{1}{2 \cosh \frac{\nu-\mu}{2}}, \quad \boldsymbol{\rho}_{\mathbf{1}}=\sqrt{\boldsymbol{Q} \boldsymbol{P} \sqrt{\boldsymbol{Q}}} \\
\left(\langle\boldsymbol{a}| \frac{1}{\sqrt{\boldsymbol{Q}}}\right)(\mu) & =e^{(a+1 / 2) \mu}, & \left(\frac{1}{\sqrt{\boldsymbol{Q}}}|\boldsymbol{l}\rangle\right)(\nu) & =e^{(l+1 / 2) \nu},
\end{aligned}
$$

then we find

$$
\begin{aligned}
\Omega(z) & =\operatorname{Det}\left(1+z\left(\boldsymbol{P}+t \sum_{p=1}^{r} \frac{1}{\sqrt{\boldsymbol{Q}}}\left|\boldsymbol{l}_{\boldsymbol{p}}\right\rangle\left\langle\boldsymbol{a}_{\boldsymbol{p}}\right| \frac{1}{\sqrt{\boldsymbol{Q}}}\right) \boldsymbol{Q}\right) \\
& =\operatorname{Det}\left(1+z \boldsymbol{\rho}_{\mathbf{1}}\right) \operatorname{det}_{p, q}\left(\delta_{p, q}+z t\left\langle\boldsymbol{a}_{\boldsymbol{p}}\left|\left(1+z \boldsymbol{\rho}_{\mathbf{1}}\right)^{-1}\right| \boldsymbol{l}_{\boldsymbol{q}}\right\rangle\right),
\end{aligned}
$$

where the multiplication among variables in the boldface character is understood as matrix multiplication with indices $\mu, \nu$ and measures in (4.7). Note that the square root $\sqrt{\boldsymbol{Q}}$ should be regarded as a formal notation. We can express the integrations without it. The reason we introduce it is because of the relation to the previous quantities as we shall see below. Here, in the last equation we have used the formula

$$
\operatorname{det}_{i, j=1, \cdots, D}\left(\delta_{i, j}+\sum_{p=1}^{r}\left(l_{p}\right)_{i}\left(a_{p}\right)_{j}\right)=\operatorname{det}_{p, q=1, \cdots, r}\left(\delta_{p, q}+\sum_{i=1}^{D}\left(a_{p}\right)_{i}\left(l_{q}\right)_{i}\right),
$$


which is the same as (4.6) if we change the variables by $(V)_{I k}=\left(l_{k}\right)_{I},\left(U^{\mathrm{T}}\right)_{k J}=\left(a_{k}\right)_{J}$. Now if we pick up the $t^{r}$ term, then we find

$$
\Xi(z)\left\langle\operatorname{det}_{p, q} W_{\left(a_{p} \mid l_{q}\right)}\left(e^{\mu}, e^{\nu}\right)\right\rangle^{\mathrm{GC}}=\operatorname{Det}\left(1+z \boldsymbol{\rho}_{\mathbf{1}}\right) \operatorname{det}_{p, q}\left\langle\boldsymbol{a}_{\boldsymbol{p}}\left|z\left(1+z \boldsymbol{\rho}_{\mathbf{1}}\right)^{-1}\right| \boldsymbol{l}_{\boldsymbol{q}}\right\rangle .
$$

This holds for both the hook and the non-hook cases.

Now using this result (4.15) we can reduce the proof of (1.6) to the result of (1.4) given in the previous section or we can prove (1.4) independently. Let us first consider to reduce to the previous result. If we pick up the constant term by taking the limit $t \rightarrow 0$, we find

$$
\sum_{N=0}^{\infty} z^{N}\langle 1\rangle_{N}=\operatorname{Det}\left(1+z \boldsymbol{\rho}_{\mathbf{1}}\right) .
$$

Comparing with the expression for the partition function (2.8), we find

$$
\operatorname{Det}\left(1+z \boldsymbol{\rho}_{1}\right)=\operatorname{Det}\left(1+z \rho_{1}\right) .
$$

Also, if we apply the above results to the single-hook case, we find

$$
\Xi(z)\left\langle W_{\left(a_{p} \mid l_{q}\right)}\left(e^{\mu}, e^{\nu}\right)\right\rangle^{\mathrm{GC}}=\operatorname{Det}\left(1+z \boldsymbol{\rho}_{\mathbf{1}}\right) z\left\langle\boldsymbol{a}_{\boldsymbol{p}}\left|\left(1+z \boldsymbol{\rho}_{\mathbf{1}}\right)^{-1}\right| \boldsymbol{l}_{\boldsymbol{q}}\right\rangle .
$$

Again comparing with the expression for the hook representation we have, we find

$$
\left\langle\boldsymbol{a}_{\boldsymbol{p}}\left|\left(1+z \boldsymbol{\rho}_{\mathbf{1}}\right)^{-1}\right| \boldsymbol{l}_{\boldsymbol{q}}\right\rangle=\left\langle a_{p}\left|\left(1+z \rho_{1}\right)^{-1}\right| l_{q}\right\rangle .
$$

Plugging (4.17) and (4.19) back to (4.15), we have shown that

$$
\left\langle W_{\left(a_{1} a_{2} \cdots a_{r} \mid l_{1} l_{1} \cdots l_{r}\right)}\right\rangle^{\mathrm{GC}}=\operatorname{det}_{p, q}\left(\left\langle a_{p}|R(z)| l_{q}\right\rangle\right) .
$$

Instead of our comparison with the known results, the argument here also suggests that if we restrict ourselves to the half BPS Wilson loop, we can have an alternative derivation for the hook case if we evaluate carefully $\operatorname{Det}\left(1+z \boldsymbol{\rho}_{\mathbf{1}}\right)$ and $\left\langle\boldsymbol{a}_{\boldsymbol{p}}\left|\left(1+z \boldsymbol{\rho}_{\mathbf{1}}\right)^{-1}\right| \boldsymbol{l}_{\boldsymbol{q}}\right\rangle$. The computation of $\operatorname{Det}\left(1+z \boldsymbol{\rho}_{\mathbf{1}}\right)$ is exactly what we did around (2.4). Also, the computation of $\left\langle\boldsymbol{a}\left|\left(1+z \boldsymbol{\rho}_{1}\right)^{-1}\right| \boldsymbol{l}\right\rangle$ becomes

$$
\int \frac{d x}{\hbar} e^{i x^{2} /(2 \hbar)} \cdots \int \frac{d y}{\hbar} e^{-i y^{2} /(2 \hbar)} e^{2 \pi\left(a+\frac{1}{2}\right) x / \hbar} \frac{e^{-i q_{x}\left(x-y^{\prime}\right) / \hbar}}{2 \cosh \frac{q_{x}}{2}} \cdots \frac{e^{-i q_{y}\left(x^{\prime}-y\right) / \hbar}}{2 \cosh \frac{q_{y}}{2}} e^{2 \pi\left(l+\frac{1}{2}\right) y / \hbar},
$$

In completing the square for $x$ and $y$ we find

$$
\begin{aligned}
\frac{i}{2 \hbar} x^{2}-\frac{i q_{x}}{\hbar} x+\frac{2 \pi(a+1 / 2)}{\hbar} x & =\frac{i}{2 \hbar}\left(x-q_{x}-2 \pi i(a+1 / 2)\right)^{2}-\frac{i}{2 \hbar}\left(q_{x}+2 \pi i(a+1 / 2)\right)^{2}, \\
-\frac{i}{2 \hbar} y^{2}+\frac{i q_{y}}{\hbar} y+\frac{2 \pi(l+1 / 2)}{\hbar} y & =-\frac{i}{2 \hbar}\left(y-q_{y}+2 \pi i(l+1 / 2)\right)^{2}+\frac{i}{2 \hbar}\left(q_{y}-2 \pi i(l+1 / 2)\right)^{2} .
\end{aligned}
$$

Note that $q^{2}$ terms cancel with the square completion from the neighboring terms. Hence, we are left with

$$
\frac{1}{2 k}\left((a+1 / 2) q_{x}+2 \pi i a(a+1)+(l+1 / 2) q_{y}-2 \pi i l(l+1)\right) .
$$

This is nothing but the exponent we found in (3.8) with (3.9). We note in passing that the above computation can be done also in the operator formalism. 


\subsection{Fermionic representation}

Our general expression (4.20) of the Wilson loop VEV suggests that there is an underlying fermionic structure. This is expected from the fermionic nature of D-branes in topological string theory [41]. Introducing the fermions

$$
\psi(x)=\sum_{n \in \mathbb{Z}} \psi_{n+\frac{1}{2}} x^{-n-1}, \quad \psi^{*}(x)=\sum_{n \in \mathbb{Z}} \psi_{n+\frac{1}{2}}^{*} x^{-n-1},
$$

with the standard anti-commutation relation

$$
\left\{\psi_{r}, \psi_{s}^{*}\right\}=\delta_{r+s, 0}
$$

such that the vacuum is annihilated by the positive modes as

$$
\psi_{r}|0\rangle=\psi_{r}^{*}|0\rangle=0 \quad \text { for } \quad r>0
$$

we define the state $|V\rangle$ as

$$
|V\rangle=\exp \left[\sum_{a, l=0}^{\infty}\left\langle W_{(a \mid l)}\right\rangle^{\mathrm{GC}} \psi_{-a-\frac{1}{2}} \psi_{-l-\frac{1}{2}}^{*}\right]|0\rangle=\exp \left[\sum_{a, l=0}^{\infty}\langle a|R(z)| l\rangle \psi_{-a-\frac{1}{2}} \psi_{-l-\frac{1}{2}}^{*}\right]|0\rangle .
$$

In terms of this state $|V\rangle$, the grand canonical VEV of the Wilson loop $W_{\left(a_{1} a_{2} \cdots a_{r} \mid l_{1} l_{2} \cdots l_{r}\right)}$ is compactly written as

$$
\left\langle W_{\left(a_{1} a_{2} \cdots a_{r} \mid l_{1} l_{1} \cdots l_{r}\right)}\right\rangle^{\mathrm{GC}}=\left\langle 0\left|\prod_{i=1}^{r} \psi_{a_{i}+\frac{1}{2}}^{*} \psi_{l_{i}+\frac{1}{2}}\right| V\right\rangle .
$$

This is reminiscent of the expression of topological vertex in [41]. Indeed, the perturbative part of a single-hook Wilson loop is determined by the topological vertex of $\mathbb{C}^{3}$

$$
\left\langle W_{(a \mid l)}\right\rangle^{\mathrm{GC}(\text { pert })}=\frac{q^{\frac{1}{4} a(a+1)-\frac{1}{4} l(l+1)}}{[a+l+1][a] ![l] !} i^{a+l+1} e^{\frac{2(a+l+1) \mu}{k}},
$$

with $[n]=q^{\frac{n}{2}}-q^{-\frac{n}{2}}$ and $q=e^{\frac{4 \pi i}{k}}$. Using the $q$-binomial formula, one can show that the alternating sum of (4.29) reproduces the perturbative part of winding Wilson loop [27]

$$
\sum_{a+l=n-1}(-1)^{l} \frac{q^{\frac{1}{4} a(a+1)-\frac{1}{4} l(l+1)}}{[a+l+1][a] ![l] !} i^{a+l+1} e^{\frac{2(a+l+1) \mu}{k}}=\frac{i^{n}}{[n]} e^{\frac{2 n \mu}{k}}=\frac{i^{n-1}}{2 \sin \frac{2 \pi}{k}} e^{\frac{2 n \mu}{k}} .
$$

\section{Relation to open topological strings}

In this section, we see a relation between the VEVs of the half BPS Wilson loops and the open topological string amplitudes. As is well-known, the ABJM matrix model is related to the $L(2,1)$ lens space matrix model by analytic continuation $[7,25]$ (see also [42-44]). This lens space matrix model is also related to the topological string on local $\mathbb{P}^{1} \times \mathbb{P}^{1}$ through the large $N$ duality [45]. In fact, the perturbative and the worldsheet instanton parts in the 
ABJM partition function can be captured by the result of the closed topological string on local $\mathbb{P}^{1} \times \mathbb{P}^{1}$. Similarly, the VEVs of the half BPS Wilson loops are described by the open topological string. Here we are interested in the VEVs in the grand canonical ensemble, which corresponds to the so-called large radius frame on the topological string side. The open topological string in this frame was recently studied in detail in [28].

We note that the membrane instanton corrections are difficult to be known from the topological string because these corrections correspond to the non-perturbative effects in the topological string. We will explore the membrane instanton corrections in the next section with the help of the numerical analysis.

First we briefly summarize the result of [28]. The open topological string amplitudes take the following general form [46-48],

$$
\begin{aligned}
F^{\text {open }}(t, V)=\sum_{\beta \in H_{2}(X)} \sum_{g=0}^{\infty} \sum_{h=1}^{\infty} & \sum_{\ell} \sum_{m=1}^{\infty} \frac{1}{h !} n_{g, \beta, \ell} \frac{1}{m}\left(2 \sinh \frac{m g_{\mathrm{top}}}{2}\right)^{2 g-2} \\
& \times \prod_{j=1}^{h}\left(\frac{2}{\ell_{j}} \sinh \frac{m \ell_{j} g_{\mathrm{top}}}{2} \operatorname{tr} V^{m \ell_{j}}\right) e^{-m \beta \cdot t},
\end{aligned}
$$

where $t$ is the Kähler moduli of the local Calabi-Yau $X$, and $V$ is the open string moduli. For the ABJM theory, we are interested in local $\mathbb{P}_{1} \times \mathbb{P}_{1}$. The string coupling in the topological string is related to the Chern-Simons level,

$$
g_{\mathrm{top}}=\frac{4 \pi i}{k} .
$$

There are two Kähler moduli, which are identified as the chemical potential $\mu$ dual to the original rank $N$,

$$
t_{1}=t_{2}=T=\frac{4 \mu}{k}-\pi i, \quad Q \equiv e^{-T}=-e^{-\frac{4 \mu}{k}} .
$$

Similarly, the open string moduli $V$ is also identified with the dual variable for the Wilson loop insertion $U$. Then, we can relate the perturbative part and worldsheet instanton part of the grand canonical VEVs in the ABJM theory to the above open topological string amplitudes. The concrete relation is given explicitly by [28],

$$
\begin{aligned}
e^{F^{\mathrm{open}}(t, \widehat{V})} & =\left\langle\exp \left[\sum_{j=1}^{\infty} \frac{1}{j} \operatorname{Str} U^{j} \operatorname{tr} V^{j}\right]\right\rangle^{\mathrm{GC}(\text { pert }+\mathrm{WS})} \\
& =\sum_{n_{1}, n_{2}, \ldots} c_{n_{1}, n_{2}, \ldots}\left\langle(\operatorname{Str} U)^{n_{1}}\left(\operatorname{Str} U^{2}\right)^{n_{2}} \ldots\right\rangle^{\mathrm{GC}(\operatorname{pert}+\mathrm{WS})}(\operatorname{tr} V)^{n_{1}}\left(\operatorname{tr} V^{2}\right)^{n_{2}} \cdots
\end{aligned}
$$

with $c_{n_{1}, n_{2}, \ldots}=1 /\left(\prod_{j} j^{n_{j}} n_{j} !\right)$. Note that to write down the relation we have to plug a new parameter

$$
\widehat{V}=Q^{-1 / 2} V=i e^{\frac{2 \mu}{k}} V
$$

into (5.1). 


\subsection{Perturbative part}

Let us consider the perturbative part. We neglect all the exponentially suppressed terms in (5.1). We observe that the leading order contribution $\beta=(0,0)$ comes only from

$$
n_{0,(0,0),(1)}=1 .
$$

Thus we obtain

$$
F_{\text {pert }}^{\text {open }}(V)=\frac{1}{i} \sum_{m=1}^{\infty} \frac{1}{m} \frac{1}{2 \sin \frac{2 \pi m}{k}} \operatorname{tr} V^{m} .
$$

Plugging this into (5.4), we get

$$
\begin{aligned}
e^{F_{\text {pert }}^{\text {open }}(\widehat{V})=} & 1+\frac{e^{\frac{2 \mu}{k}}}{2 \sin \frac{2 \pi}{k}} \operatorname{tr} V+\frac{e^{\frac{4 \mu}{k}}}{8 \sin ^{2} \frac{2 \pi}{k}}(\operatorname{tr} V)^{2}+\frac{i e^{\frac{4 \mu}{k}}}{4 \sin \frac{4 \pi}{k}} \operatorname{tr} V^{2} \\
& +\frac{e^{\frac{6 \mu}{k}}}{48 \sin ^{3} \frac{2 \pi}{k}}(\operatorname{tr} V)^{3}+\frac{i e^{\frac{6 \mu}{k}}}{8 \sin \frac{2 \pi}{k} \sin \frac{4 \pi}{k}} \operatorname{tr} V \operatorname{tr} V^{2}-\frac{e^{\frac{6 \mu}{k}}}{6 \sin \frac{6 \pi}{k}} \operatorname{tr} V^{3}+\cdots
\end{aligned}
$$

Therefore we immediately find

$$
\left\langle\operatorname{Str} U^{n}\right\rangle^{\mathrm{GC}(\text { pert })}=\frac{i^{n-1}}{2 \sin \frac{2 \pi n}{k}} e^{\frac{2 n \mu}{k}},
$$

and the factorization property

$$
\left\langle(\operatorname{Str} U)^{n_{1}}\left(\operatorname{Str} U^{2}\right)^{n_{2}} \cdots\right\rangle^{\mathrm{GC}(\text { pert })}=\left(\langle\operatorname{Str} U\rangle^{\mathrm{GC}(\text { pert })}\right)^{n_{1}}\left(\left\langle\operatorname{Str} U^{2}\right\rangle^{\mathrm{GC}(\text { pert })}\right)^{n_{2}} \cdots .
$$

Note that this factorization property does not hold if the instanton effect is taken into account. One can check that these results reproduce (4.29) for the hook representations.

From the factorization property (5.10), one finds that the perturbative part of the half BPS Wilson loop in the representation $\mathbf{R}$ scales as

$$
\left\langle W_{\mathbf{R}}\right\rangle^{\mathrm{GC}(\text { pert })} \sim e^{\frac{2 n \mu}{k}},
$$

where $n$ is the number of boxes of Young diagram $\mathbf{R}$ and we have dropped the prefactor independent of $\mu$. Coming back to the VEV in the canonical ensemble via (2.20), we find that the perturbative part of the half BPS Wilson loop in arbitrary representation gives the following Airy function behavior

$$
\left\langle W_{\mathbf{R}}\right\rangle_{N}^{(\text {pert })} \sim \operatorname{Ai}\left[\left(\frac{2}{\pi^{2} k}\right)^{-1 / 3}\left(N-\frac{k}{24}-\frac{6 n+1}{3 k}\right)\right],
$$

where the proportional coefficient depends only on $k$. From this expression, we can also find the large $N$ limit as

$$
\frac{\left\langle W_{\mathbf{R}}\right\rangle_{N}}{Z(N)} \sim e^{n \pi \sqrt{2 \lambda}} \quad(N \rightarrow \infty),
$$

where $\lambda=N / k$ is the 't Hooft coupling. Note that this exponent is the same as $n$ times of an classical string action on the gravity side [24]. 


\subsection{Worldsheet instantons}

Let us consider the worldsheet instanton corrections. We first denote the general open string amplitude by

$$
F^{\text {open }}(t, V)=\sum_{h=1}^{\infty} \sum_{\ell} \sum_{m=1}^{\infty} \mathcal{A}_{\ell_{1}, \ldots, \ell_{h}}^{(m)} \operatorname{tr} V^{m \ell_{1}} \cdots \operatorname{tr} V^{m \ell_{h}},
$$

with

$$
\mathcal{A}_{\ell_{1}, \ldots, \ell_{h}}^{(m)}=\sum_{\beta} \sum_{g=0}^{\infty} \frac{1}{h !} n_{g, \beta, \ell} \frac{1}{m}\left(2 \sinh \frac{m g_{\mathrm{top}}}{2}\right)^{2 g-2} \prod_{j=1}^{h}\left(\frac{2}{\ell_{j}} \sinh \frac{m \ell_{j} g_{\mathrm{top}}}{2}\right) e^{-m \beta \cdot t} .
$$

After specifying $\beta=\left(d_{1}, d_{2}\right)$ and take the "diagonal" sum for the open GV invariants

$$
n_{g, d, \ell}=\sum_{d_{1}+d_{2}=d} n_{g,\left(d_{1}, d_{2}\right), \ell}
$$

this becomes

$$
\mathcal{A}_{\ell_{1}, \ldots, \ell_{h}}^{(m)}=\sum_{d=0}^{\infty} \sum_{g=0}^{\infty} \frac{(-1)^{g-1}}{h !} n_{g, d, \ell} \frac{1}{m}\left(2 \sin \frac{2 \pi m}{k}\right)^{2 g-2} \prod_{j=1}^{h}\left(\frac{2 i}{\ell_{j}} \sin \frac{2 \pi m \ell_{j}}{k}\right) Q^{m d} .
$$

Thus we find from (5.14), for example,

$$
\begin{aligned}
\langle\mathrm{Str} U\rangle^{\mathrm{GC}(\text { pert+WS })} \operatorname{tr} V & =\mathcal{A}_{1}^{(1)} \operatorname{tr} \widehat{V} \\
\frac{1}{2}\left\langle\operatorname{Str} U^{2}\right\rangle^{\mathrm{GC}(\text { pert+WS })} \operatorname{tr} V^{2} & =\left(\mathcal{A}_{2}^{(1)}+\mathcal{A}_{1}^{(2)}\right) \operatorname{tr} \widehat{V}^{2}, \\
\frac{1}{2}\left\langle(\mathrm{Str} U)^{2}\right\rangle^{\mathrm{GC}(\text { pert+WS })}(\operatorname{tr} V)^{2} & =\left(\mathcal{A}_{1,1}^{(1)}+\frac{1}{2}\left(\mathcal{A}_{1}^{(1)}\right)^{2}\right)(\operatorname{tr} \widehat{V})^{2},
\end{aligned}
$$

where the relation between $V$ and $\widehat{V}$ is given by (5.5).

Using the explicit values of the open GV invariants listed in tables 1 and 2 of [28], we obtain the worldsheet instanton corrections up to order $Q^{5}$,

$$
\begin{aligned}
& \langle\operatorname{Str} U\rangle^{\mathrm{GC}(\text { pert }+\mathrm{WS})}=\frac{e^{\frac{2 \mu}{k}}}{2 \sin \frac{2 \pi}{k}}\left[1+2 Q+3 Q^{2}+10 Q^{3}+\left(49-32 \sin ^{2} \frac{2 \pi}{k}\right) Q^{4}\right. \\
& \left.\quad+\left(288-576 \sin ^{2} \frac{2 \pi}{k}+352 \sin ^{4} \frac{2 \pi}{k}\right) Q^{5}+\mathcal{O}\left(Q^{6}\right)\right] \\
& \left\langle\mathrm{Str} U^{2}\right\rangle^{\mathrm{GC}(\text { pert }+\mathrm{WS})}=\frac{i e^{\frac{4 \mu}{k}}}{\sin \frac{4 \pi}{k} \sin ^{2} \frac{2 \pi}{k}}\left[\frac{1}{2} \sin ^{2} \frac{2 \pi}{k}+\frac{1}{2} \sin ^{2} \frac{4 \pi}{k} Q\right. \\
& \quad+\left(\sin ^{2} \frac{2 \pi}{k}+\sin ^{2} \frac{4 \pi}{k}\right) Q^{2}+4 \sin ^{2} \frac{4 \pi}{k} Q^{3} \\
& \quad+\left(\frac{3}{2} \sin ^{2} \frac{2 \pi}{k}+18 \sin ^{2} \frac{4 \pi}{k}-14 \sin ^{2} \frac{2 \pi}{k} \sin ^{2} \frac{4 \pi}{k}\right) Q^{4} \\
& \left.\quad+\left(104-224 \sin ^{2} \frac{2 \pi}{k}+160 \sin ^{4} \frac{2 \pi}{k}\right) \sin ^{2} \frac{4 \pi}{k} Q^{5}+\mathcal{O}\left(Q^{6}\right)\right],
\end{aligned}
$$




$$
\begin{aligned}
& \left\langle(\operatorname{Str} U)^{2}\right\rangle^{\mathrm{GC}(\text { pert }+\mathrm{WS})}=\frac{e^{\frac{4 \mu}{k}}}{\sin ^{2} \frac{2 \pi}{k}}\left[\frac{1}{4}+\left(1-\sin ^{2} \frac{2 \pi}{k}\right) Q+\left(\frac{5}{2}-2 \sin ^{2} \frac{2 \pi}{k}\right) Q^{2}\right. \\
& +\left(8-8 \sin ^{2} \frac{2 \pi}{k}\right) Q^{3}+\left(\frac{147}{4}-64 \sin ^{2} \frac{2 \pi}{k}+28 \sin ^{4} \frac{2 \pi}{k}\right) Q^{4} \\
& \left.\quad+\left(208-656 \sin ^{2} \frac{2 \pi}{k}+768 \sin ^{4} \frac{2 \pi}{k}-320 \sin ^{6} \frac{2 \pi}{k}\right) Q^{5}+\mathcal{O}\left(Q^{6}\right)\right]
\end{aligned}
$$

As discussed in [49], the $g=0$ terms of $\langle\operatorname{Str} U\rangle^{\mathrm{GC}(\text { pert+WS) }}$ are given by the factor $(Q / z)^{\frac{1}{2}}$ representing the worldsheet instanton corrections to the disk amplitude. Here $z$ and $Q$ are related by the mirror map of local $\mathbb{P}^{1} \times \mathbb{P}^{1}$ along the diagonal slice $z_{1}=z_{2}=z$

$$
\frac{1}{2} \log \frac{Q}{z}=2 z_{4} F_{3}\left(1,1, \frac{3}{2}, \frac{3}{2} ; 2,2,2 ; 16 z\right) .
$$

Inverting this relation, the worldsheet instanton corrections to the disk amplitude are found to be

$$
\begin{aligned}
f(Q) & \equiv\left(\frac{Q}{z}\right)^{\frac{1}{2}} \\
& =1+2 Q+3 Q^{2}+10 Q^{3}+49 Q^{4}+288 Q^{5}+1892 Q^{6}+13390 Q^{7}+\cdots
\end{aligned}
$$

which reproduce the invariants $n_{g=0, d,(1)}$ listed in [28]. Interstingly, we find from [48] that the VEV of the Wilson loop with widing $n$ is generically written as the following form,

$$
\left\langle\operatorname{Str} U^{n}\right\rangle^{\mathrm{GC}(\text { pert+WS })}=e^{\frac{2 n \mu}{k}} f\left(Q^{n}\right) \sum_{g, d} \sum_{n=\ell m} N_{\vec{e}_{m}, d}^{g}\left(2 \sin \frac{2 \pi \ell}{k}\right)^{2 g-2}\left(2 \sin \frac{2 \pi n}{k}\right) Q^{d \ell}
$$

where $N_{\vec{e}_{m}, d}^{g}$ are integers, which are related to the open GV invariants $n_{g, d, \ell}$. Instead of the topological string consideration, we can also fix such integers by comparing the matrix model results $[7,25,27]$ in the 't Hooft limit because the genus expansion in this limit captures all the worldsheet instanton corrections. The similar comparison on the worldsheet instanton corrections to the grand potential has been done in [18]. In this way, we have fixed the values of $N_{\vec{e}_{m}, d}^{g}$ in the very first few cases. The result is summarized in table 1 . For $n=1,2$, one can check that (5.22) with table 1 indeed reproduce (5.19).

In the next section, we will confirm that these worldsheet instanton corrections are indeed consistent with our numerical results.

\section{$6 \quad$ Numerical study and membrane instantons}

In this section, we numerically evaluate the VEVs of the half BPS Wilson loops in hook representations by using the formulation presented in sections 3 and 4 . The main motivation of this analysis is to explore the membrane instanton effects, which are very hard to be described in the topological string theory. The similar analysis has been already done for the grand partition function in $[18,20]$. We compute the VEVs in various hook representations for some values of $k$. Here we propose that the membrane instanton corrections are completely encoded by the replacement $\mu \rightarrow \mu_{\text {eff }}$ in the perturbative part and 


\begin{tabular}{|rrrrccc|}
\hline$N_{\vec{e}_{1}, d}^{g}$ & $d=0$ & 1 & 2 & 3 & 4 & 5 \\
\hline$g=0$ & 1 & 0 & 0 & 0 & 0 & 0 \\
1 & 0 & 0 & 0 & 0 & -8 & -128 \\
2 & 0 & 0 & 0 & 0 & 0 & 22 \\
\hline
\end{tabular}

\begin{tabular}{|rrrrr|}
\hline$N_{\vec{e}_{2}, d}^{g}$ & $d=0$ & 1 & 2 & 3 \\
\hline$g=0$ & 0 & 1 & 2 & 6 \\
1 & 0 & 0 & 0 & 0 \\
\hline
\end{tabular}

\begin{tabular}{|rrrrr|}
\hline$N_{\vec{e}_{3}, d}^{g}$ & $d=0$ & 1 & 2 & 3 \\
\hline$g=0$ & 0 & 1 & 3 & 9 \\
1 & 0 & 0 & 0 & 0 \\
\hline
\end{tabular}

\begin{tabular}{|rrrcc|}
\hline$N_{\vec{e}_{4}, d}^{g}$ & $d=0$ & 1 & 2 & 3 \\
\hline$g=0$ & 0 & 1 & 4 & 14 \\
1 & 0 & 0 & -4 & -8 \\
\hline
\end{tabular}

Table 1. The values of $N_{\vec{e}_{m}, d}^{g}$.

the worldsheet instanton part as in (1.8). The effective chemical potential $\mu_{\text {eff }}$ is explicitly given [20] for even $k=2 n$ as

$$
\mu_{\mathrm{eff}}=\mu+(-1)^{n-1} 2 e^{-2 \mu}{ }_{4} F_{3}\left(1,1, \frac{3}{2}, \frac{3}{2} ; 2,2,2 ;(-1)^{n} 16 e^{-2 \mu}\right),
$$

and conjectured for odd $k$ as

$$
\mu_{\mathrm{eff}}=\mu+e^{-4 \mu}{ }_{4} F_{3}\left(1,1, \frac{3}{2}, \frac{3}{2} ; 2,2,2 ;-16 e^{-4 \mu}\right) .
$$

Below, we will check the proposal (1.8) by the numerical study.

\subsection{A procedure}

Let us consider the VEV for the half BPS Wilson loops in the hook representation $(a \mid l)$. The VEV is given by (1.4),

$$
\left\langle W_{(a \mid l)}\right\rangle^{\mathrm{GC}}=\int \frac{d x d y}{(2 \pi k)^{2}}\langle a \mid x\rangle\left\langle x\left|\frac{z}{1+z \rho_{1}}\right| y\right\rangle\langle y \mid l\rangle .
$$

Let us first note that, the complex phase dependence only come from $\langle a \mid x\rangle$ and $\langle y \mid l\rangle$, which is trivially given in (3.9),

$$
\left\langle W_{(a \mid l)}\right\rangle^{\mathrm{GC}}=e^{\frac{a(a+1) \pi i}{k}-\frac{l(l+1) \pi i}{k}}\left|\left\langle W_{(a \mid l)}\right\rangle^{\mathrm{GC}}\right| .
$$

Hence we define a real function $\mathcal{W}_{(a \mid l)}$ with its series expansion $\mathcal{W}_{(a \mid l)}^{(m)}$ as

$$
\mathcal{W}_{(a \mid l)} \equiv\left|\left\langle W_{(a \mid l)}\right\rangle^{\mathrm{GC}}\right|=\sum_{m=0}^{\infty}(-1)^{m} z^{m+1} \mathcal{W}_{(a \mid l)}^{(m)}
$$

where $\mathcal{W}_{(a \mid l)}^{(m)}$ is given by

$$
\mathcal{W}_{(a \mid l)}^{(m)}=\int \frac{d x d y}{(2 \pi k)^{2}} \widehat{f}_{a}(x) \rho_{1}^{m}(x, y) \widehat{f}_{l}(y), \quad \widehat{f}_{n}(x) \equiv \frac{e^{\left(n+\frac{1}{2}\right) \frac{x}{k}}}{\sqrt{2 \cosh \frac{x}{2}}} .
$$


Of course, the VEVs for $(a \mid l)$ and $(l \mid a)$ should be complex conjugate to each other, therefore we immediately find

$$
\mathcal{W}_{(a \mid l)}=\mathcal{W}_{(l \mid a)}
$$

Our task is to evaluate the integral (6.6). This can be done as follows. Let us introduce the function by

$$
\phi_{l}^{(m)}(x)=\frac{1}{\sqrt{2 \cosh \frac{x}{2}}} \int \frac{d y}{2 \pi k} \rho_{1}^{m}(x, y) \widehat{f}_{l}(y)
$$

One easily finds that this function satisfies the recurrence relation

$$
\phi_{l}^{(m)}(x)=\frac{1}{2 \cosh \frac{x}{2}} \int \frac{d y}{2 \pi k} \frac{1}{2 \cosh \frac{x-y}{2 k}} \phi_{l}^{(m-1)}(y),
$$

with the initial condition

$$
\phi_{l}^{(0)}(x)=\frac{e^{\left(l+\frac{1}{2}\right) \frac{x}{k}}}{2 \cosh \frac{x}{2}} .
$$

Once the function $\phi_{l}^{(m)}(x)$ is known, the integral (6.6) is easily evaluated as

$$
\mathcal{W}_{(a \mid l)}^{(m)}=\int \frac{d x}{2 \pi k} e^{\left(a+\frac{1}{2}\right) \frac{x}{k}} \phi_{l}^{(m)}(x)
$$

We notice that the integral equation (6.9) is essentially the same as that appearing in [1618]. One can solve it for any $k$ at least numerically. Practically, we solve the integral equation up to a certain value $m=m_{\max }$, and make an approximation for $\mathcal{W}_{(a \mid l)}(6.5)$ as

$$
\mathcal{W}_{(a \mid l)} \approx \sum_{m=0}^{m_{\max }}(-1)^{m} z^{m+1} \mathcal{W}_{(a \mid l)}^{(m)}
$$

Though this approximation is originally valid only for the small $\mu$ regime, we extrapolate the profile to a reasonably large $\mu$ regime and fit the expansion coefficients of $\mathcal{W}_{(a \mid l)}$ by exact values at this regime. This is the same strategy as that in [18].

Before closing this subsection, we will briefly comment on the convergence of integral. In (6.10) and (6.11), there appear the exponential factors that diverge in large $x$ limit. Due to these factors, the integral (6.11) converges only if

$$
k>2(a+l+1)=2\left|\mathbf{R}_{\mathrm{hook}}\right|,
$$

where $\left|\mathbf{R}_{\text {hook }}\right|$ is the size of Young diagram corresponding to the hook representation $\mathbf{R}_{\text {hook }}$. Therefore, the grand canonical VEVs, and correspondingly the canonical VEVs $\left\langle W_{\mathbf{R}}\right\rangle_{N}$, are well-defined only for such values of $k$, though it is much harder to see it directly in $\left\langle W_{\mathbf{R}}\right\rangle_{N}$ due to the complex phases in the original expression (1.2). Such a behavior has also been found for the multiple winding Wilson loop in [27]. 


\subsection{Fundamental representation}

The simplest representation is the fundamental representation $(0 \mid 0)$,

$$
\left\langle W_{(0 \mid 0)}\right\rangle^{\mathrm{GC}}=\mathcal{W}_{(0 \mid 0)}
$$

We would like to evaluate $\mathcal{W}_{(0 \mid 0)}$ numerically for some values of $k$. By solving the integral equation, we have performed the numerical computation, and find the non-perturbative corrections to $\mathcal{W}_{(0 \mid 0)}$ for $k=3,4,6,8,12$. The results are as follows:

$$
\begin{aligned}
& \left.\mathcal{W}_{(0 \mid 0)}\right|_{k=3}=\mathcal{W}_{(0 \mid 0)}^{(\text {pert })}\left(1+2 Q+3 Q^{2}+\frac{28}{3} Q^{3}+\frac{79}{3} Q^{4}+60 Q^{5}+\frac{1562}{9} Q^{6}+\mathcal{O}\left(Q^{7}\right)\right), \\
& \left.\mathcal{W}_{(0 \mid 0)}\right|_{k=4}=\mathcal{W}_{(0 \mid 0)}^{(\text {pert })}\left(1+2 Q+2 Q^{2}+12 Q^{3}+22 Q^{4}+124 Q^{5}+276 Q^{6}+\mathcal{O}\left(Q^{7}\right)\right), \\
& \mathcal{W}_{\left.(0 \mid 0)\right|_{k=6}}=\mathcal{W}_{(0 \mid 0)}^{(\text {pert })}\left(1+2 Q+3 Q^{2}+\frac{28}{3} Q^{3}+\frac{79}{3} Q^{4}+60 Q^{5}+\frac{1562}{9} Q^{6}+\mathcal{O}\left(Q^{7}\right)\right), \\
& \mathcal{W}_{\left.(0 \mid 0)\right|_{k=8}}=\mathcal{W}_{(0 \mid 0)}^{(\text {pert })}\left(1+2 Q+3 Q^{2}+10 Q^{3}+\frac{65}{2} Q^{4}+89 Q^{5}+\frac{465}{2} Q^{6}+\mathcal{O}\left(Q^{7}\right)\right), \\
& \left.\mathcal{W}_{(0 \mid 0)}\right|_{k=12}=\mathcal{W}_{(0 \mid 0)}^{(\text {pert })}\left(1+2 Q+3 Q^{2}+10 Q^{3}+41 Q^{4}+166 Q^{5}+\frac{1844}{3} Q^{6}+\mathcal{O}\left(Q^{7}\right)\right),
\end{aligned}
$$

where $\mathcal{W}_{(0 \mid 0)}^{(\text {pert })}$ is

$$
\mathcal{W}_{(0 \mid 0)}^{(\mathrm{pert})}=\frac{e^{\frac{2 \mu}{k}}}{2 \sin \frac{2 \pi}{k}} .
$$

To obtain the numerical results, we have chosen $m_{\max }$ to be the best value of the numerical fitting at each instanton number. This best value decreases as the instanton number increases because of the exponential suppression of the corrections and the numerical errors. Anyway, for all the above cases, we have chosen $m_{\max } \sim 10$, and the numerical results of the coefficients match to these exact values (6.15) in about 5-digit accuracy. Especially, if we make a wrong guess at a certain instanton order, the next order of instanton would grow exponentially, which could make our fitting totally impossible. Note that for the above values of $k$ the coefficients of (6.16) become particularly simple (especially rational) because the root of unity $e^{2 \pi i / k}$ takes the simple values. This is why we choose the above values of $k$ for our fitting problem.

Let us compare these results with the theoretical prediction. The worldsheet instanton corrections of $\left\langle W_{(0 \mid 0)}\right\rangle^{\mathrm{GC}}=\langle\operatorname{Str} U\rangle^{\mathrm{GC}}$ are given by (5.19). As mentioned before, we propose that the membrane instanton correction can be incorporated by the replacement $\mu \rightarrow \mu_{\text {eff }}$ in the worldsheet instanton correction. Thus our conjecture, including the membrane instanton effects, is

$$
\begin{gathered}
\mathcal{W}_{(0 \mid 0)}=\frac{e^{\frac{2 \mu_{\mathrm{eff}}}{k}}}{2 \sin \frac{2 \pi}{k}}\left[1+2 Q_{\mathrm{eff}}+3 Q_{\mathrm{eff}}^{2}+10 Q_{\mathrm{eff}}^{3}+\left(49-32 \sin ^{2} \frac{2 \pi}{k}\right) Q_{\mathrm{eff}}^{4}\right. \\
\left.+\left(288-576 \sin ^{2} \frac{2 \pi}{k}+352 \sin ^{4} \frac{2 \pi}{k}\right) Q_{\mathrm{eff}}^{5}+\mathcal{O}\left(Q_{\mathrm{eff}}^{6}\right)\right],
\end{gathered}
$$


where $Q_{\text {eff }}=-e^{-\frac{4 \mu_{\text {eff }}}{k}}$. For the comparison, we need to rewrite it in terms of $Q=-e^{-\frac{4 \mu}{k}}$. Using the relations (6.1) and (6.2) between $\mu$ and $\mu_{\text {eff }}$, we find

$$
Q_{\text {eff }}= \begin{cases}Q+\frac{4}{3} Q^{4}+\mathcal{O}\left(Q^{7}\right) & (k=3,6) \\ Q+2 Q^{3}+11 Q^{5}+\mathcal{O}\left(Q^{7}\right) & (k=4) \\ Q+Q^{5}+\mathcal{O}\left(Q^{9}\right) & (k=8) \\ Q+\frac{2}{3} Q^{7}+\mathcal{O}\left(Q^{13}\right) & (k=12) .\end{cases}
$$

Plugging these into (6.17), one can check that the corrections exactly agree with the numerical ones (6.15) up to $Q^{5}$. We emphasize that only the worldsheet instanton correction does not explain the numerical results (6.15). We need to replace $\mu$ by $\mu_{\text {eff }}$ to reproduce them. This is due to the membrane instanton effects.

\subsection{Young diagrams with two boxes}

There are two representations with two-box Young diagrams:

$$
\left\langle W_{(1 \mid 0)}\right\rangle^{\mathrm{GC}}=e^{\frac{2 \pi i}{k}} \mathcal{W}_{(1 \mid 0)}, \quad\left\langle W_{(0 \mid 1)}\right\rangle^{\mathrm{GC}}=e^{-\frac{2 \pi i}{k}} \mathcal{W}_{(0 \mid 1)}
$$

We have the relation $\mathcal{W}_{(1 \mid 0)}=\mathcal{W}_{(0 \mid 1)}$. By the similar computation to the fundamental representation, we find

$$
\begin{aligned}
\left.\mathcal{W}_{(1 \mid 0)}\right|_{k=6} & =\mathcal{W}_{(1 \mid 0)}^{\text {(pert) }}\left(1+Q+4 Q^{2}+\frac{20}{3} Q^{3}+18 Q^{4}+\frac{172}{3} Q^{5}+\frac{1190}{9} Q^{6}+\mathcal{O}\left(Q^{7}\right)\right), \\
\left.\mathcal{W}_{(1 \mid 0)}\right|_{k=8} & =\mathcal{W}_{(1 \mid 0)}^{(\text {pert })}\left(1+2 Q+6 Q^{2}+16 Q^{3}+46 Q^{4}+128 Q^{5}+364 Q^{6}+\mathcal{O}\left(Q^{7}\right)\right), \\
\left.\mathcal{W}_{(1 \mid 0)}\right|_{k=12} & =\mathcal{W}_{(1 \mid 0)}^{\text {(pert) }}\left(1+3 Q+8 Q^{2}+24 Q^{3}+90 Q^{4}+348 Q^{5}+\frac{3862}{3} Q^{6}+\mathcal{O}\left(Q^{7}\right)\right),
\end{aligned}
$$

with

$$
\mathcal{W}_{(1 \mid 0)}^{(\text {pert })}=\frac{e^{\frac{4 \mu}{k}}}{4 \sin \frac{2 \pi}{k} \sin \frac{4 \pi}{k}} .
$$

Let us also compare these results with our prediction. Note that $\left\langle W_{(1 \mid 0)}\right\rangle^{\mathrm{GC}}$ is given by

$$
\left\langle W_{(1 \mid 0)}\right\rangle^{\mathrm{GC}}=\frac{1}{2}\left\langle(\operatorname{Str} U)^{2}\right\rangle^{\mathrm{GC}}+\frac{1}{2}\left\langle\operatorname{Str} U^{2}\right\rangle^{\mathrm{GC}} .
$$

The worldsheet instanton corrections of $\left\langle(\operatorname{Str} U)^{2}\right\rangle^{\mathrm{GC}}$ and $\left\langle\operatorname{Str} U^{2}\right\rangle^{\mathrm{GC}}$ are given by (5.19). Thus our prediction is

$$
\begin{aligned}
\mathcal{W}_{(1 \mid 0)} & =\frac{e^{\frac{4 \mu_{\mathrm{eff}}}{k}}}{4 \sin ^{3} \frac{2 \pi}{k} \sin \frac{4 \pi}{k}}\left[\sin ^{2} \frac{2 \pi}{k}+\sin ^{2} \frac{4 \pi}{k} Q_{\mathrm{eff}}+\left(2 \sin ^{2} \frac{2 \pi}{k}+2 \sin ^{2} \frac{4 \pi}{k}\right) Q_{\mathrm{eff}}^{2}\right. \\
& +8 \sin ^{2} \frac{4 \pi}{k} Q_{\mathrm{eff}}^{3}+\left(3 \sin ^{2} \frac{2 \pi}{k}+36 \sin ^{2} \frac{4 \pi}{k}-28 \sin ^{2} \frac{2 \pi}{k} \sin ^{2} \frac{4 \pi}{k}\right) Q_{\mathrm{eff}}^{4} \\
& \left.+\left(208-448 \sin ^{2} \frac{2 \pi}{k}+320 \sin ^{4} \frac{2 \pi}{k}\right) \sin ^{2} \frac{4 \pi}{k} Q_{\mathrm{eff}}^{5}+\mathcal{O}\left(Q_{\mathrm{eff}}^{6}\right)\right] .
\end{aligned}
$$

Using the relation (6.18), one finds that the corrections again agree with the numerical ones (6.20) up to $Q^{5}$. 


\subsection{Young diagrams with three boxes}

For the three-box Young diagrams, there are two non-trivial real functions,

$$
\left\langle W_{(2 \mid 0)}\right\rangle^{\mathrm{GC}}=e^{\frac{6 \pi i}{k}} \mathcal{W}_{(2 \mid 0)}, \quad\left\langle W_{(1 \mid 1)}\right\rangle^{\mathrm{GC}}=\mathcal{W}_{(1 \mid 1)}, \quad\left\langle W_{(0 \mid 2)}\right\rangle^{\mathrm{GC}}=e^{-\frac{6 \pi i}{k}} \mathcal{W}_{(0 \mid 2)},
$$

with the constraint $\mathcal{W}_{(2 \mid 0)}=\mathcal{W}_{(0 \mid 2)}$. From the numerical analysis, we find

$$
\begin{aligned}
\left.\mathcal{W}_{(2 \mid 0)}\right|_{k=8} & =\mathcal{W}_{(2 \mid 0)}^{(\text {pert })}\left(1+Q^{2}+8 Q^{3}+\frac{33}{2} Q^{4}+40 Q^{5}+\frac{235}{2} Q^{6}+\mathcal{O}\left(Q^{7}\right)\right), \\
\left.\mathcal{W}_{(2 \mid 0)}\right|_{k=12} & =\mathcal{W}_{(2 \mid 0)}^{(\text {pert })}\left(1+2 Q+8 Q^{2}+32 Q^{3}+116 Q^{4}+426 Q^{5}+1534 Q^{6}+\mathcal{O}\left(Q^{7}\right)\right),
\end{aligned}
$$

and

$$
\begin{gathered}
\left.\mathcal{W}_{(1 \mid 1)}\right|_{k=8}=\mathcal{W}_{(1 \mid 1)}^{(\text {pert })}\left(1+2 Q+5 Q^{2}+14 Q^{3}+\frac{73}{2} Q^{4}+105 Q^{5}+\frac{591}{2} Q^{6}+\mathcal{O}\left(Q^{7}\right)\right), \\
\left.\mathcal{W}_{(1 \mid 1)}\right|_{k=12}=\mathcal{W}_{(1 \mid 1)}^{(\text {pert })}\left(1+4 Q+12 Q^{2}+38 Q^{3}+136 Q^{4}+508 Q^{5}+1866 Q^{6}+\mathcal{O}\left(Q^{7}\right)\right) .
\end{gathered}
$$

Note that to compare these results with the theoretical prediction, we need to know the open GV invariants $n_{g, d, \ell}$ for $\boldsymbol{\ell}=(3),(2,1),(1,1,1)$, whose explicit values are not found in the literature. Instead, one can compare the result for the Wilson loop with winding 3. The VEV of the Wilson loop with winding 3 is computed as

$$
\begin{aligned}
\left\langle W_{3}\right\rangle^{\mathrm{GC}} & =\left\langle W_{(2 \mid 0)}\right\rangle^{\mathrm{GC}}-\left\langle W_{(1 \mid 1)}\right\rangle^{\mathrm{GC}}+\left\langle W_{(0 \mid 2)}\right\rangle^{\mathrm{GC}} \\
& =2 \cos \left(\frac{6 \pi}{k}\right) \mathcal{W}_{(2 \mid 0)}-\mathcal{W}_{(1 \mid 1)} .
\end{aligned}
$$

From (5.22) with table 1, on the other hand, we obtain

$$
\begin{aligned}
\left\langle W_{3}\right\rangle^{\mathrm{GC}}= & -e^{\frac{6 \mu_{\mathrm{eff}}}{k}}\left[\frac{1}{2} \csc \frac{6 \pi}{k}+\frac{1}{2} \csc ^{2} \frac{2 \pi}{k} \sin \frac{6 \pi}{k} Q_{\mathrm{eff}}+\frac{3}{2} \csc ^{2} \frac{2 \pi}{k} \sin \frac{6 \pi}{k} Q_{\mathrm{eff}}^{2}\right. \\
& \left.+\left(\csc \frac{6 \pi}{k}+\frac{9}{2} \csc ^{2} \frac{2 \pi}{k} \sin \frac{6 \pi}{k}\right) Q_{\mathrm{eff}}^{3}+\mathcal{O}\left(Q_{\mathrm{eff}}^{4}\right)\right] .
\end{aligned}
$$

One can check that this reproduces the above results for $k=8,12$ up to $Q^{3}$.

\subsection{Young diagrams with four boxes}

For the four-box case, there are four hook representations and one non-hook representation. For the hook representations, we have

$$
\begin{array}{ll}
\left\langle W_{(3 \mid 0)}\right\rangle^{\mathrm{GC}}=e^{\frac{12 \pi i}{k}} \mathcal{W}_{(3 \mid 0)}, & \left\langle W_{(2 \mid 1)}\right\rangle^{\mathrm{GC}}=e^{\frac{4 \pi i}{k}} \mathcal{W}_{(2 \mid 1)}, \\
\left\langle W_{(0 \mid 3)}\right\rangle^{\mathrm{GC}}=e^{-\frac{12 \pi i}{k}} \mathcal{W}_{(0 \mid 3)}, & \left\langle W_{(1 \mid 2)}\right\rangle^{\mathrm{GC}}=e^{-\frac{4 \pi i}{k}} \mathcal{W}_{(1 \mid 2)},
\end{array}
$$

with $\mathcal{W}_{(3 \mid 0)}=\mathcal{W}_{(0 \mid 3)}$ and $\mathcal{W}_{(2 \mid 1)}=\mathcal{W}_{(1 \mid 2)}$. For the non-hook representation $(1,0 \mid 1,0)$, the $\mathrm{VEV}$ is given by the determinant formula

$$
\left\langle W_{(1,0 \mid 1,0)}\right\rangle^{\mathrm{GC}}=\operatorname{det}\left(\begin{array}{c}
\left\langle W_{(1 \mid 1)}\right\rangle^{\mathrm{GC}}\left\langle W_{(1 \mid 0)}\right\rangle^{\mathrm{GC}} \\
\left\langle W_{(0 \mid 1)}\right\rangle^{\mathrm{GC}}\left\langle W_{(0 \mid 0)}\right\rangle^{\mathrm{GC}}
\end{array}\right)=\mathcal{W}_{(1 \mid 1)} \mathcal{W}_{(0 \mid 0)}-\mathcal{W}_{(1 \mid 0)} \mathcal{W}_{(0 \mid 1)}
$$


From the numerical analysis, we find

$$
\begin{aligned}
& \left.\mathcal{W}_{(3 \mid 0)}\right|_{k=12}=\mathcal{W}_{(3 \mid 0)}^{(\text {pert })}\left(1+Q^{2}+12 Q^{3}+61 Q^{4}+216 Q^{5}+\frac{1417}{2} Q^{6}+\mathcal{O}\left(Q^{7}\right)\right), \\
& \left.\mathcal{W}_{(2 \mid 1)}\right|_{k=12}=\mathcal{W}_{(2 \mid 1)}^{\text {(pert) }}\left(1+3 Q+10 Q^{2}+36 Q^{3}+133 Q^{4}+486 Q^{5}+\frac{5258}{3} Q^{6}+\mathcal{O}\left(Q^{7}\right)\right) .
\end{aligned}
$$

One can check that the VEV of the Wilson loop with winding 4 at $k=12$ is reproduced from these results.

\subsection{Implications}

The grand canonical VEVs of the half BPS Wilson loops are in general complex. As was seen before, however, their phase dependences are trivial. This fact implies that there are some non-trivial relation among open GV invariants $n_{g, d, \ell}$ for different $\boldsymbol{\ell}$. Let us see this here. In the size 2 representations, we have

$$
\begin{gathered}
\left\langle(\operatorname{Str} U)^{2}\right\rangle^{\mathrm{GC}}=\left\langle W_{(1 \mid 0)}\right\rangle^{\mathrm{GC}}+\left\langle W_{(0 \mid 1)}\right\rangle^{\mathrm{GC}}=2 \cos \frac{2 \pi}{k} \mathcal{W}_{(1 \mid 0)}, \\
\left\langle\operatorname{Str} U^{2}\right\rangle^{\mathrm{GC}}=\left\langle W_{(1 \mid 0)}\right\rangle^{\mathrm{GC}}-\left\langle W_{(0 \mid 1)}\right\rangle^{\mathrm{GC}}=2 i \sin \frac{2 \pi}{k} \mathcal{W}_{(1 \mid 0)},
\end{gathered}
$$

where we have used $\mathcal{W}_{(1 \mid 0)}=\mathcal{W}_{(0 \mid 1)}$. These expressions immediately lead to the exact relation,

$$
\frac{\left\langle\operatorname{Str} U^{2}\right\rangle^{\mathrm{GC}}}{\left\langle(\operatorname{Str} U)^{2}\right\rangle^{\mathrm{GC}}}=i \tan \frac{2 \pi}{k}
$$

This relation gives a non-trivial relation among the open GV invariants $n_{g, d,(1)}, n_{g, d,(2)}$ and $n_{g, d,(1,1)}$. For very lower $g$ and $d$, we find

$$
\begin{aligned}
& n_{0,1,(2)}=n_{0,1,(1,1)}=\frac{n_{0,1,(1)}}{2} \\
& n_{0,2,(2)}=n_{0,2,(1,1)}=\frac{1}{4}\left(2 n_{0,2,(1)}+n_{0,1,(1)}^{2}-n_{0,1,(1)}\right) \\
& n_{0,3,(2)}=n_{0,3,(1,1)}=\frac{1}{2}\left(n_{0,3,(1)}+n_{0,2,(1)} n_{0,1,(1)}\right) \\
& n_{0,4,(2)}=\frac{1}{4}\left(2 n_{0,4,(1)}+2 n_{0,3,(1)} n_{0,1,(1)}+n_{0,2,(1)}^{2}-n_{0,2,(1)}\right) \\
& n_{0,4,(1,1)}-4 n_{1,4,(1,1)}=\frac{1}{4}\left(2 n_{0,4,(1)}+2 n_{0,3,(1)} n_{0,1,(1)}+n_{0,2,(1)}^{2}-n_{0,2,(1)}-8 n_{1,4,(1)}\right), \\
& n_{1,4,(1,1)}=n_{1,4,(2)} .
\end{aligned}
$$

One can check that the expressions (5.19) indeed satisfy the relation (6.33) up to order $Q^{5}$.

Similarly, from the relation

$$
\begin{aligned}
\frac{1}{3}\left\langle(\operatorname{Str} U)^{3}\right\rangle^{\mathrm{GC}}+\frac{2}{3}\left\langle\operatorname{Str} U^{3}\right\rangle^{\mathrm{GC}} & =2 \cos \frac{6 \pi}{k} \mathcal{W}_{(2 \mid 0)}, \\
\left\langle\operatorname{Str} U \operatorname{Str} U^{2}\right\rangle^{\mathrm{GC}} & =2 i \sin \frac{6 \pi}{k} \mathcal{W}_{(2 \mid 0)}
\end{aligned}
$$


we find

$$
\left\langle\operatorname{Str} U \operatorname{Str} U^{2}\right\rangle^{\mathrm{GC}}=i \tan \frac{6 \pi}{k}\left(\frac{1}{3}\left\langle(\operatorname{Str} U)^{3}\right\rangle^{\mathrm{GC}}+\frac{2}{3}\left\langle\operatorname{Str} U^{3}\right\rangle^{\mathrm{GC}}\right) .
$$

This gives an non-trivial relation among $n_{g, d, \ell}$ for $\boldsymbol{\ell}=(1),(2),(1,1),(3),(2,1),(1,1,1)$.

Also, the Giambelli formula (1.6) gives non-trivial relations among the open GV invariants. For the representation $\mathbf{R}=(1,0 \mid 1,0)$, we find the relation

$$
\begin{aligned}
& \frac{1}{12}\left\langle(\operatorname{Str} U)^{4}\right\rangle^{\mathrm{GC}}-\frac{1}{3}\left\langle\operatorname{Str} U \mathrm{Str} U^{3}\right\rangle^{\mathrm{GC}}+\frac{1}{4}\left\langle\left(\mathrm{Str} U^{2}\right)^{2}\right\rangle^{\mathrm{GC}} \\
& =\frac{1}{3}\left\langle(\mathrm{Str} U)^{3}\right\rangle^{\mathrm{GC}}\langle\operatorname{Str} U\rangle^{\mathrm{GC}}-\frac{1}{4}\left(\left\langle(\operatorname{Str} U)^{2}\right\rangle^{\mathrm{GC}}\right)^{2} \\
& -\frac{1}{3}\langle\operatorname{Str} U\rangle^{\mathrm{GC}}\left\langle\operatorname{Str} U^{3}\right\rangle^{\mathrm{GC}}+\frac{1}{4}\left(\left\langle\operatorname{Str} U^{2}\right\rangle^{\mathrm{GC}}\right)^{2},
\end{aligned}
$$

or equivalently, in terms of $\mathcal{A}_{\ell_{1}, \ldots, \ell_{h}}^{(m)}$ defined by (5.17), the relation is written as

$$
\mathcal{A}_{1,1,1,1}^{(1)}-\mathcal{A}_{3,1}^{(1)}+\mathcal{A}_{2,2}^{(1)}=-\mathcal{A}_{1,1}^{(2)}-\left(\mathcal{A}_{1,1}^{(1)}\right)^{2} .
$$

Substituting (5.17) into this, we obtain the relation among the open GV invariants. ${ }^{7}$

\section{Conclusion}

In this paper we have proposed the Fermi gas formalism for the VEVs of the half BPS Wilson loops in arbitrary representations. For the case of the hook representations, we present the formula in terms of the convolution of integrations. For the case of the non-hook representations, we reduce the computation to the hook case by a determinant formula similar to the Giambelli formula for the Schur polynomial. After working out these expressions for the VEVs, we also present a numerical study. We find that besides the worldsheet instanton corrections we also have the membrane instanton corrections which can be incorporated by shifting the chemical potential $\mu$ into $\mu_{\text {eff }}$ as we did in studying the bound states in the ABJM partition function.

We conclude our paper by listing several discussions on the further directions.

Based on the numerical results, we conclude that the membrane instanton correction is completely encoded in the perturbative and the worldsheet instanton parts by replacing $\mu$ by $\mu_{\text {eff }}$. Let us recall that in the partition function, there is also a pure membrane instanton correction, as well as the bound states of the worldsheet instantons and the membrane instantons. This pure membrane instanton correction is directly related to the non-perturbative effect in the closed topological string [21] (see also [50]). Our Wilson loop result (1.8) implies that there seem to be no pure membrane instanton corrections in the open topological string on "diagonal" local $\mathbb{P}^{1} \times \mathbb{P}^{1}$. It would be interesting to confirm this in the topological string framework.

\footnotetext{
${ }^{7}$ To capture the membrane instanton correction, we need to replace $Q$ in (5.17) by $Q_{\text {eff }}$, but this replacement does not change the relations at all.
} 
Most of our analysis here focus on the half BPS Wilson loops, which have nice counterparts in the topological string. Our method presented in section 2, however, can be applicable to the 1/6 BPS Wilson loops. The topological string counterparts to such 1/6 BPS Wilson loops are unclear, thus it would be important to reveal the structure of instanton effects in the 1/6 BPS Wilson loops by using our method. It is also interesting to perform Monte Carlo simulation [51] of the 1/6 BPS Wilson loops in low dimensional representations, which has been useful for the partition function $[14,15]$. It would also be illuminating to apply our formalism to other observables in the ABJM theory such as the vortex loop [6] and energymomentum tensor correlator [52], which can be also simplified by the localization method.

In the topological string theory, we have a set of open GV invariants for each representation. In our Fermi gas formalism, we find several non-trivial relations among such invariants. The simplest one is the symmetry of taking the transpose in the Young diagram. For example, disregarding a difference in the trivial phase factor, the VEVs of the half BPS ABJM Wilson loops in the symmetric and anti-symmetric representations are equal with each other. Hence this triviality of the phase factor imposes highly non-trivial relations in the open GV invariants. The origin of this property is unclear on the topological string side at present. Besides, the VEVs in the non-hook representations enjoy the Giambelli property. Technically, the Giambelli property imposes many interesting relations and reduces largely the unknown open GV invariants. Using the Giambelli property, we can show that the number of unknown GV invariants at each order reduces to the number of boxes $n$, which originally increases with the number of representations, namely, partitions $p(n) \sim e^{\pi \sqrt{\frac{2 n}{3}}} /(4 \sqrt{3} n)$. It is interesting to clarify what kind of relations the transposition symmetry and the Giambelli compatibility will impose on the open GV invariants. We also ask whether these kinds of relations appear in more general topological string theories or not. Since we have studied only the local $\mathbb{P}^{1} \times \mathbb{P}^{1}$ topological string, the relations might be accidental properties in this model. If these are common in a class of topological strings, we expect that there are some extra structures, which naturally explain the relations. For example, since the topological recursion of Eynard and Orantin [53] gives relations among all open string invariants, this might explain the relations coming from the transposition symmetry and the Giambelli compatibility.

A natural open question is the physical interpretation of the Giambelli compatibility. It would be nice to understand its meaning from the brane configuration or the gravity analysis. We hope that this would be a clue to understand M-theory.

\section{Acknowledgments}

We are grateful to Heng-Yu Chen, Nadav Drukker, Marcos Marino, Tomoki Nosaka, Kazutoshi Ohta, Soo-Jong Rey, Masaki Shigemori, Takao Suyama for useful discussions. Especially we would like to thank Sho Matsomoto for sharing his idea of the proof of Giambelli compatibity with us. The work of Y.H. is supported in part by the JSPS Research Fellowship for Young Scientists, while the work of K.O. is supported in part by JSPS Grant-in-Aid for Young Scientists (B) \#23740178. 
Open Access. This article is distributed under the terms of the Creative Commons Attribution License which permits any use, distribution and reproduction in any medium, provided the original author(s) and source are credited.

\section{References}

[1] O. Aharony, O. Bergman, D.L. Jafferis and J. Maldacena, $N=6$ superconformal Chern-Simons-matter theories, M2-branes and their gravity duals, JHEP 10 (2008) 091 [arXiv:0806.1218] [INSPIRE].

[2] V. Pestun, Localization of gauge theory on a four-sphere and supersymmetric Wilson loops, Commun. Math. Phys. 313 (2012) 71 [arXiv:0712.2824] [INSPIRE].

[3] A. Kapustin, B. Willett and I. Yaakov, Exact results for Wilson loops in superconformal Chern-Simons theories with matter, JHEP 03 (2010) 089 [arXiv:0909.4559] [INSPIRE].

[4] D.L. Jafferis, The exact superconformal R-symmetry extremizes Z, JHEP 05 (2012) 159 [arXiv: 1012.3210] [INSPIRE].

[5] N. Hama, K. Hosomichi and S. Lee, Notes on SUSY gauge theories on three-sphere, JHEP 03 (2011) 127 [arXiv:1012.3512] [INSPIRE].

[6] N. Drukker, T. Okuda and F. Passerini, Exact results for vortex loop operators in $3 d$ supersymmetric theories, arXiv:1211.3409 [INSPIRE].

[7] N. Drukker, M. Mariño and P. Putrov, From weak to strong coupling in ABJM theory, Commun. Math. Phys. 306 (2011) 511 [arXiv:1007.3837] [InSPIRE].

[8] N. Drukker, M. Mariño and P. Putrov, Nonperturbative aspects of ABJM theory, JHEP 11 (2011) 141 [arXiv:1103.4844] [INSPIRE].

[9] H. Fuji, S. Hirano and S. Moriyama, Summing up all genus free energy of ABJM matrix model, JHEP 08 (2011) 001 [arXiv:1106.4631] [INSPIRE].

[10] M. Mariño and P. Putrov, ABJM theory as a Fermi gas, J. Stat. Mech. 03 (2012) P03001 [arXiv:1110.4066] [INSPIRE].

[11] C.P. Herzog, I.R. Klebanov, S.S. Pufu and T. Tesileanu, Multi-matrix models and tri-Sasaki Einstein spaces, Phys. Rev. D 83 (2011) 046001 [arXiv: 1011.5487] [INSPIRE].

[12] K. Okuyama, A note on the partition function of ABJM theory on $S^{3}$, Prog. Theor. Phys. 127 (2012) 229 [arXiv:1110.3555] [INSPIRE].

[13] A. Kapustin, B. Willett and I. Yaakov, Nonperturbative tests of three-dimensional dualities, JHEP 10 (2010) 013 [arXiv: 1003.5694] [INSPIRE].

[14] M. Hanada et al., Numerical studies of the ABJM theory for arbitrary $N$ at arbitrary coupling constant, JHEP 05 (2012) 121 [arXiv:1202.5300] [INSPIRE].

[15] M. Honda et al., Monte Carlo studies of $3 d N=6$ SCFT via localization method, PoS (LATTICE 2012) 233 [arXiv: 1211.6844] [INSPIRE].

[16] Y. Hatsuda, S. Moriyama and K. Okuyama, Exact results on the ABJM Fermi gas, JHEP 10 (2012) 020 [arXiv:1207.4283] [INSPIRE].

[17] P. Putrov and M. Yamazaki, Exact ABJM partition function from TBA, Mod. Phys. Lett. A 27 (2012) 1250200 [arXiv:1207.5066] [INSPIRE]. 
[18] Y. Hatsuda, S. Moriyama and K. Okuyama, Instanton effects in ABJM theory from Fermi gas approach, JHEP 01 (2013) 158 [arXiv:1211.1251] [INSPIRE].

[19] F. Calvo and M. Mariño, Membrane instantons from a semiclassical TBA, JHEP 05 (2013) 006 [arXiv:1212.5118] [INSPIRE].

[20] Y. Hatsuda, S. Moriyama and K. Okuyama, Instanton bound states in ABJM theory, JHEP 05 (2013) 054 [arXiv: 1301.5184] [INSPIRE].

[21] Y. Hatsuda, M. Mariño, S. Moriyama and K. Okuyama, Non-perturbative effects and the refined topological string, arXiv:1306.1734 [INSPIRE].

[22] A. Cagnazzo, D. Sorokin and L. Wulff, String instanton in $A d S_{4} \times C P^{3}$, JHEP 05 (2010) 009 [arXiv:0911.5228] [INSPIRE].

[23] K. Becker, M. Becker and A. Strominger, Five-branes, membranes and nonperturbative string theory, Nucl. Phys. B 456 (1995) 130 [hep-th/9507158] [INSPIRE].

[24] N. Drukker and D. Trancanelli, A supermatrix model for $N=6$ super Chern-Simons-matter theory, JHEP 02 (2010) 058 [arXiv:0912.3006] [INSPIRE].

[25] M. Mariño and P. Putrov, Exact results in ABJM theory from topological strings, JHEP 06 (2010) 011 [arXiv:0912.3074] [INSPIRE].

[26] I. Bars, Supergroups and their representations, Lectures Appl. Math. 21 (1983) 17 [INSPIRE].

[27] A. Klemm, M. Mariño, M. Schiereck and M. Soroush, ABJM Wilson loops in the Fermi gas approach, arXiv:1207.0611 [INSPIRE].

[28] A. Grassi, J. Kallen and M. Mariño, The topological open string wavefunction, arXiv: 1304.6097 [INSPIRE].

[29] M.S. Bianchi, G. Giribet, M. Leoni and S. Penati, The 1/2 BPS Wilson loop in ABJM theory at two loops, Phys. Rev. D 88 (2013) 026009 [arXiv:1303.6939] [InSPIRE].

[30] D. Farquet and J. Sparks, Wilson loops and the geometry of matrix models in $A d S_{4} / C F T_{3}$, arXiv: 1304.0784 [INSPIRE].

[31] N. Kim, Supersymmetric Wilson loops with general contours in ABJM theory, Mod. Phys. Lett. A 28 (2013) 1350150 [arXiv:1304.7660] [INSPIRE].

[32] L. Griguolo, D. Marmiroli, G. Martelloni and D. Seminara, The generalized cusp in ABJ(M) $N=6$ super Chern-Simons theories, JHEP 05 (2013) 113 [arXiv:1208.5766] [INSPIRE].

[33] V. Cardinali, L. Griguolo, G. Martelloni and D. Seminara, New supersymmetric Wilson loops in $A B J(M)$ theories, Phys. Lett. B 718 (2012) 615 [arXiv:1209.4032] [InSPIRE].

[34] T. Suyama, A systematic study on matrix models for Chern-Simons-matter theories, Nucl. Phys. B 874 (2013) 528 [arXiv:1304.7831] [INSPIRE].

[35] A. Borodin, G. Olshanski and E. Strahov, Giambelli compatible point processes, Adv. Appl. Math. 37.2 (2006) 209 [math-ph/0505021].

[36] N. Drukker, J. Plefka and D. Young, Wilson loops in 3-dimensional $N=6$ supersymmetric Chern-Simons theory and their string theory duals, JHEP 11 (2008) 019 [arXiv:0809.2787] [INSPIRE].

[37] B. Chen and J.-B. Wu, Supersymmetric Wilson loops in $N=6$ super Chern-Simons-matter theory, Nucl. Phys. B $\mathbf{8 2 5}$ (2010) 38 [arXiv:0809.2863] [inSPIRE]. 
[38] S.-J. Rey, T. Suyama and S. Yamaguchi, Wilson loops in superconformal Chern-Simons theory and fundamental strings in anti-de Sitter supergravity dual, JHEP 03 (2009) 127 [arXiv:0809.3786] [INSPIRE].

[39] G. Olshanski, A. Regev and A. Vershik, Frobenius-Schur functions, math.C0/0110077.

[40] E.M. Moens and J. Van der Jeugt, A determinantal formula for supersymmetric Schur polynomials, J. Alg. Comb. 17.3 (2003) 283.

[41] M. Aganagic, R. Dijkgraaf, A. Klemm, M. Mariño and C. Vafa, Topological strings and integrable hierarchies, Commun. Math. Phys. 261 (2006) 451 [hep-th/0312085] [INSPIRE].

[42] R. Dijkgraaf and C. Vafa, $N=1$ supersymmetry, deconstruction and bosonic gauge theories, hep-th/0302011 [INSPIRE].

[43] R. Dijkgraaf, S. Gukov, V.A. Kazakov and C. Vafa, Perturbative analysis of gauged matrix models, Phys. Rev. D 68 (2003) 045007 [hep-th/0210238] [INSPIRE].

[44] H. Awata, S. Hirano and M. Shigemori, The partition function of ABJ theory, Prog. Theor. Exp. Phys. (2013) 053B04 [arXiv:1212.2966] [INSPIRE].

[45] M. Aganagic, A. Klemm, M. Mariño and C. Vafa, Matrix model as a mirror of Chern-Simons theory, JHEP 02 (2004) 010 [hep-th/0211098] [INSPIRE].

[46] H. Ooguri and C. Vafa, Knot invariants and topological strings, Nucl. Phys. B 577 (2000) 419 [hep-th/9912123] [INSPIRE].

[47] J. Labastida, M. Mariño and C. Vafa, Knots, links and branes at large- $N$, JHEP 11 (2000) 007 [hep-th/0010102] [INSPIRE].

[48] M. Mariño and C. Vafa, Framed knots at large-N, Contemp. Math. 310 (2002) 185 [hep-th/0108064] [INSPIRE].

[49] M. Aganagic, A. Klemm and C. Vafa, Disk instantons, mirror symmetry and the duality web, Z. Naturforsch. A 57 (2002) 1 [hep-th/0105045] [INSPIRE].

[50] G. Lockhart and C. Vafa, Superconformal partition functions and non-perturbative topological strings, arXiv:1210.5909 [INSPIRE].

[51] M. Hanada, M. Honda, Y. Honma, J. Nishimura, S. Shiba and Y. Yoshida, in preparation.

[52] C. Closset, T.T. Dumitrescu, G. Festuccia and Z. Komargodski, Supersymmetric field theories on three-manifolds, JHEP 05 (2013) 017 [arXiv:1212.3388] [INSPIRE].

[53] B. Eynard and N. Orantin, Invariants of algebraic curves and topological expansion, Commun. Num. Theor. Phys. 1 (2007) 347 [math-ph/0702045] [INSPIRE]. 